\title{
High-Resolution Passive SAR Imaging Exploiting Structured Bayesian Compressive Sensing
}

\author{
Qisong Wu, Member, IEEE, Yimin D. Zhang, Senior Member, IEEE, \\ Moeness G. Amin, Fellow, IEEE, and Braham Himed, Fellow, IEEE
}

\begin{abstract}
In this paper, we develop a novel structured Bayesian compressive sensing algorithm with location dependence for high-resolution imaging in ultra-narrowband passive synthetic aperture radar (SAR) systems. The proposed technique exploits wide-angle and/or multi-angle observations for image resolution enhancement. We first introduce a forward model based on sparse synthetic apertures. The problem of sparse scatterer imaging is formulated as an optimization problem of reconstructing group sparse signals. A logistic Gaussian kernel model, which involves a logistic model and location-dependent Gaussian kernel, and takes the correlation between entire scatterers into account, is then used to encourage the underlying continuity structure of illuminated target scene in a nonparametric Bayesian learning framework. The posterior inference of the proposed method is then provided in the Markov Chain Monte Carlo (MCMC) sampling scheme. The proposed technique enables high-resolution SAR imaging in wide-angle as well as multi-angle observation systems, and the imaging performance is improved by exploiting the underlying structure of the target scene. Simulation and experiment results demonstrate the superiority of the proposed algorithms in preserving the continuous structure and suppressing isolated components over existing state-of-the-art compressive sensing methods.
\end{abstract}

Index Terms-Passive radar, synthetic aperture radar (SAR), wide-angle imaging, multiple-angle imaging, Bayesian compressive sensing

\section{INTRODUCTION}

Radar imaging, such as synthetic aperture radar (SAR) and inverse SAR (ISAR), has been widely used in active radar systems [1]. In recent years, passive radar systems, which utilize broadcast, navigation, and communication signals as sources of opportunity, have attracted significant interests due to their low cost, covertness, and availability of rich illuminator sources $[2,3]$.

Copyright (c) 2015 IEEE. Personal use of this material is permitted. However, permission to use this material for any other purposes must be obtained from the IEEE by sending a request to pubs-permissions @ieee.org.

The work of Q. Wu, Y. D. Zhang, and M. G. Amin was supported in part by a subcontract with Defense Engineering Corporation for research sponsored by the Air Force Research Laboratory under Contract FA8650-12-D-1376.

Q. Wu was with the Center for Advanced Communications, Villanova University, Villanova, PA 19085, USA. He is now with the Key Laboratory of Underwater Acoustic Signal Processing of Ministry of Education, Southeast University, Nanjing 210096, China (email: qisong.wu@ seu.edu.cn).

Y. D. Zhang was with the Center for Advanced Communications, Villanova University, Villanova, PA 19085, USA. He is now with the Department of Electrical and Computer Engineering, College of Engineering, Temple University, Philadelphia, PA 19122, USA (email: ydzhang@temple.edu).

M. G. Amin is with the Center for Advanced Communications, Villanova University, Villanova, PA 19085, USA.

B. Himed is with the RF Technology Branch, Air Force Research Lab (AFRL/RYMD), WPAFB, OH 45433, USA.
Passive radars differ from active radar systems in a number of aspects, such as the extremely narrow signal bandwidth, low operating frequency, low signal-to-noise ratio (SNR), and bistatic or multistatic operations [2-4]. From a radar imaging perspective, both the low operating frequency and extremely narrow signal bandwidth are adverse factors to high-resolution imaging, causing coarse range resolutions [57]. For example, a Digital Video Broadcasting-Terrestrial (DVB-T) channel uses a $7.8 \mathrm{MHz}$ bandwidth in the 450$900 \mathrm{MHz}$ band. The corresponding bistatic range resolution is approximately $20 \mathrm{~m}$ when applying conventional Fourierbased methods, such as the filtered backprojection (FBP) [8] and direct Fourier reconstruction (DFR) [9]. This figure is far below the submeter resolution achieved by typical active SAR imaging systems. Resolution enhancement can be achieved via spectrum synthesis techniques [10-12] and spectrum estimation-based techniques [13]. Fortunately, spectrum diversity is also a trait of passive radar systems due to the presence of diverse illuminator sources, i.e., the receivers can be designed to acquire a wide range of spectrum such that the image resolution can be enhanced [14]. However, unlike the wide continuous spectrum available in active SAR/ISAR systems, the resulting spectrum in a passive radar usually assumes a sparse support consisting of multiple disconnected sub-spectra depending on the available sources of opportunity and the geometries of the transmitter-receiver pairs [5, 7]. This property lends itself to high sidelobes and artifacts in Fourierbased imaging methods.

High-resolution techniques based on regularized optimization have shown effective in active SAR/ISAR imaging [1518]. The main idea is to formulate radar imaging as a sparse signal recovery problem. The orthogonal matching pursuit (OMP) [19] and Lasso [20] algorithms are widely used regularized optimization tools for effective sparse signal reconstruction. Direct applications of these methods assume invariant scattering coefficients during the radar beam illumination due to a narrow observation aperture $[15,16]$. This assumption is reasonable in active SAR/ISAR imaging systems because of their high operating frequencies. For example, to achieve a 1$\mathrm{m}$ azimuth resolution, an observation angle of about $0.86^{\circ}$ is required in the X-band SAR system, whereas this requirement becomes $20^{\circ}$ in a passive radar exploiting DVB-T signals. According to the radar backscattering principle, invariant scattering coefficients in such a wide observation aperture is impractical [21]. This becomes more pronounced in multiangle observation systems involving multiple distributed transmitters (illuminators) and/or multiple distributed receivers. In such multi-angle scenarios, the scattering coefficients of 
targets are different due to the diverse observation angles.

Given a sparse scene, we utilize the fact that the support of sparse scatterers, referred in the two-dimensional (2-D) scene coordinates, is identical or at least highly overlaps across different observation angles. As such, we can make use of such group sparsity (also referred to as block sparsity) of the scatterers for significant reconstruction performance enhancement [22, 23]. Bayesian compressive sensing (BCS) techniques are effective tools for sparse signal reconstruction [24-28]. BCS methods have been successfully applied in microwave imaging, direction finding, space-time adaptive processing, diagnosis of large linear array, and time-frequency analysis [29-36]. The multi-task CS (mt-CS) algorithm [27], which solves group sparse problems in the BCS context, was originally designed to recover real-valued sparse entries as encountered in, for example, image and video processing. The mt-CS was extended to solve complex-valued problems which are typical in radar applications, such as direction finding and radar imaging [29, 30, 36-38]. These methods exploit the fact that the real and imaginary components of a complex-valued sparse entry share the same support and, thereby, achieve improved performance and robustness to noise.

The aforementioned algorithms, however, do not consider the structure of the scatterer scene. In practical radar imaging applications, different signal structures may be exploited to facilitate the sparse reconstruction and enhance the image quality. For example, scatterers considered in the SAR/ISAR imaging often exhibit clustered structures, which can be used to enhance the imaging quality [39-42]. In these methods, the correlation of scatterers are considered over a small local region, such as the first-order neighboring scatterers as discussed in $[39,41,42]$ and the extended second-order neighboring scatterers as investigated in [39]. These approaches designate a number of structured patterns where favorable priors are placed to encourage a subset of patterns whereas the other patterns are discouraged. In real-world applications, the signal patterns do not necessarily match such designated patterns, thereby leading to sparse reconstruction performance loss. The Boltzmann machine, a commonly used graphical model, is introduced to model the general structure correlation between pixels, and massage passing and mean-field approximation techniques are, respectively introduced to perform inference [43, 44]. Dynamic CS technique is proposed to capture both amplitude and support correlation structure of timevarying signals [45]. However, these methods do not take the group sparsity into account induced by wide-angle/multi-angle observations.

In this paper, we develop a novel structure-aware BCS algorithm to acquire enhanced high-resolution images for wide-angle and/or multi-angle passive radar systems. While we focus our discussion on passive radar SAR imaging, the proposed approach can be used for ISAR imaging as well. The proposed method can also be applied to other radar applications, such as active SAR and through-thewall radar imaging. In the problem being considered in this paper, we assume that the signals of opportunity used for radar imaging is extremely narrowband, and wide-angle and/or multi-angle observations are utilized to achieve high- resolution imaging. The scatterers are considered to have spatially extended structures. To encourage the continuity of the reconstructed target scene, the proposed BCS algorithm adopts a novel logistic Gaussian kernel, which involves a logistic model and location-dependence Gaussian kernel, to account for the correlation among scatterers with locationdependent weighting coefficients. The subsequent posterior inference is implemented based on Markov Chain Monte Carlo (MCMC) sampling scheme. The concept of the logistic Gaussian kernel was first presented in our earlier work [46]. In this paper, we further extend the proposed logistic Gaussian kernel model into the wide-angle/multi-angle SAR imaging problem, where the underlying target structure within each subaperture as well as the group sparsity across a wide aperture are taken into account. As such, the proposed technique provides a powerful means for high-resolution passive radar imaging from a synthetic aperture formed from wide-angle and/or multi-angle observations with multi-static transmitter/receiver pairs. The wide-angle and/or multi-angle observations are group sparse, i.e., they correspond to the same scatterer position support but the exact scattering coefficients are generally aspect-dependent. The proposed structure-aware BCS technique utilizes both continuous target structure as well as the group sparsity due to wide-angle/multi-angle observations.

The remainder of the paper is organized as follows. Section II introduces a forward model in the passive imaging system. Section III describes the proposed structured BCS approach for effective reconstruction of sparse signals with the underlying structure. Subsequently, the posterior inference based on MCMC sampling scheme is provided in Section IV. Simulation and synthetic experiment results are presented in Section V to verify the effectiveness of the proposed algorithm. Finally, Section VI concludes this paper.

Notations: We use lower-case (upper-case) bold characters to represent vectors (matrices). In particular, $\mathbf{I}_{N}$ denotes the $N \times N$ identity matrix. (.) represents complex conjugation, whereas $(.)^{T}$ and $(.)^{H}$, respectively, denote the transpose and conjugate transpose of a matrix or vector. $\operatorname{diag}(\mathbf{x})$ represents a diagonal matrix that uses the elements of $\mathbf{x}$ as its diagonal elements, and $\|\cdot\|$ implies the Euclidean $\left(l_{2}\right)$ norm of a vector. $\mathrm{p}(\cdot)$ expresses the probability density function (pdf). $\mathcal{N}(x \mid a, b)$ and $\mathcal{C N}(x \mid a, b)$, respectively, denote that random variable $x$ follows real and complex Gaussian distributions with mean $a$ and variance $b . \operatorname{Gamma}(x \mid a, b)$ denotes that random variable $x$ follows a Gamma distribution with parameters $a$ and $b$. $\operatorname{Bern}(x \mid \pi)$ implies that random variable $x$ follows a Bernoulli distribution with weight $\pi$, and $\operatorname{PG}(x \mid a, b)$ represents the Polya-Gamma (PG) distribution with parameters $a$ and $b$. $\mathbb{E}(\cdot)$ denotes the statistical expectation operation, and $\delta(x)$ represents the Dirac delta function of $x$. We use $\circ$ to denote element-wise multiplication of two vectors.

\section{FORWARD MODEL}

Consider a wide-angle passive SAR system that senses a sparse scene, as depicted in Fig. 1. The receiver, which is mounted on an airborne platform, has the capability of 


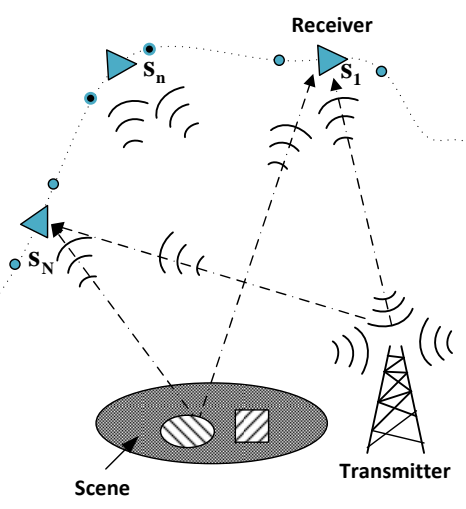

Fig. 1. Geometry of a passive sparse SAR system.

receiving reflected signals from the targets and direct-path signals from the transmitter.

Denote the position of the stationary transmitter as $\mathbf{t}$, and the $n$th azimuth location of the moving receiver as $\mathbf{s}_{n}, n=$ $1, \cdots, N$. The received signal is expressed by [47],

$$
\begin{aligned}
f\left(t, \mathbf{s}_{n}, \mathbf{t}\right) & \approx \int \exp \left(-j \omega\left[t-\frac{R\left(\mathbf{s}_{n}, \mathbf{x}, \mathbf{t}\right)}{c}\right]\right) \\
& \cdot \omega^{2} \hat{p}(\omega) \eta\left(\mathbf{x}, \mathbf{s}_{n}\right) A\left(\omega, \mathbf{x}, \mathbf{s}_{n}\right) d \omega d \mathbf{x},
\end{aligned}
$$

where $t$ denotes time, $c$ is the speed of light, $R\left(\mathbf{s}_{n}, \mathbf{x}, \mathbf{t}\right)=$ $\left\|\mathbf{x}-\mathbf{s}_{n}\right\|+\|\mathbf{x}-\mathbf{t}\|$ is the bistatic range, and $\eta\left(\mathbf{x}, \mathbf{s}_{n}\right)$ is the scattering amplitude of target at position $\mathbf{x}$ when the receiver is positioned at $\mathbf{s}_{n}$. In addition, $\hat{p}(\omega)$ is the Fourier transform of the transmitted waveform $p(t), \omega$ is the transmitted radian frequency, and $A\left(\omega, \mathbf{x}, \mathbf{s}_{n}\right)$ is given by

$$
A\left(\omega, \mathbf{x}, \mathbf{s}_{n}\right)=\frac{J_{R}\left(\omega, \mathbf{x}, \mathbf{s}_{n}\right) \cdot J_{T}(\omega, \mathbf{x}, \mathbf{t})}{(2 \pi)^{2}\left\|\mathbf{x}-\mathbf{s}_{n}\right\| \cdot\|\mathbf{x}-\mathbf{t}\|}
$$

where $J_{T}(\omega, \mathbf{x}, \mathbf{t})$ and $J_{R}\left(\omega, \mathbf{x}, \mathbf{s}_{n}\right)$ are the transmit and receive antenna beam patterns, respectively. For narrowband waveforms, the carrier frequency $\omega_{0}$ is used to replace $\omega$ in Eq. (1), and the received signal (1) can be rewritten as [48, 49],

$$
\begin{aligned}
f\left(t, \mathbf{s}_{n}, \mathbf{t}\right) & =\int \exp \left[-j \omega_{0}\left(t-\frac{R\left(\mathbf{s}_{n}, \mathbf{x}, \mathbf{t}\right)}{c}\right)\right] \\
& \cdot \tilde{p}\left(t-\frac{R\left(\mathbf{s}_{n}, \mathbf{x}, \mathbf{t}\right)}{c}\right) \eta\left(\mathbf{x}, \mathbf{s}_{n}\right) A\left(\omega_{0}, \mathbf{x}, \mathbf{s}_{n}\right) d \mathbf{x}
\end{aligned}
$$

where $\tilde{p}(t)$ is the slowly time-varying complex envelope of $p(t)$.

The direct-path (reference) waveform is expressed as

$$
\begin{aligned}
f_{\mathrm{ref}}\left(t, \mathbf{s}_{n}, \mathbf{t}\right) & =\exp \left(-j \omega_{0}\left[t-\frac{\left\|\mathbf{s}_{n}-\mathbf{t}\right\|}{c}\right]\right) \\
& \cdot \tilde{p}\left(t-\frac{\left\|\mathbf{s}_{n}-\mathbf{t}\right\|}{c}\right) \cdot \frac{J_{R}\left(\omega_{0}, \mathbf{s}_{n}\right) \cdot J_{T}\left(\omega_{0}, \mathbf{t}\right)}{(2 \pi)^{2}\left\|\mathbf{s}_{n}-\mathbf{t}\right\|},
\end{aligned}
$$

where $J_{T}\left(\omega_{0}, \mathbf{t}\right)$ and $J_{R}\left(\omega_{0}, \mathbf{s}_{n}\right)$ are the transmit and receive antenna gains. We assume that the direct-path reference signal transmitted from each illuminator is perfectly reconstructed free of multipath scattering and contamination from targets and other objects. Such reference signal reconstruction can be achieved by receiving a clean direct-path signal through a directional antenna or an array beam toward the respective transmitters, and minimizing the propagation error using the error correction coding scheme. These are standard assumptions commonly used for passive radar signal processing [4, 50].

We use the reference waveform $f_{\text {ref }}\left(t, \mathbf{s}_{n}, \mathbf{t}\right)$ to perform matched filtering of $f\left(t, \mathbf{s}_{n}, \mathbf{t}\right)$, resulting in

$$
\begin{gathered}
g\left(\tau, \mathbf{s}_{n}, \mathbf{t}\right)=\int f\left(t, \mathbf{s}_{n}, \mathbf{t}\right) \overline{f_{\text {ref }}\left(t-\tau, \mathbf{s}_{n}, \mathbf{t}\right)} d t \\
=\int C_{0} \psi_{n}\left(\tau, \mathbf{x}, \mathbf{s}_{n}, \mathbf{t}\right) w_{n}(\mathbf{x}) d \mathbf{x},
\end{gathered}
$$

where $\tau$ is the sampling delay that corresponds to the range,

$$
C_{0}=\frac{\overline{J_{R}\left(\omega_{0}, \mathbf{s}_{n}\right)} \cdot \overline{J_{T}\left(\omega_{0}, \mathbf{t}\right)}}{(2 \pi)^{2}\left\|\mathbf{s}_{n}-\mathbf{t}\right\|}
$$

is a scalar which is independent of the illuminated scene, and

$$
\psi_{n}\left(\tau, \mathbf{x}, \mathbf{s}_{n}, \mathbf{t}\right)=\chi\left(\tau, \mathbf{x}, \mathbf{s}_{n}, \mathbf{t}\right) \exp \left(-j \omega_{0} \eta_{n}(\mathbf{x})\right)
$$

is the observation kernel at position $\mathbf{s}_{n}$. In addition,

$\chi\left(\tau, \mathbf{x}, \mathbf{s}_{n}, \mathbf{t}\right)=\int \tilde{p}\left(t-\frac{R\left(\mathbf{s}_{n}, \mathbf{x}, \mathbf{t}\right)}{c}\right) \overline{\tilde{p}\left(t-\tau-\frac{\left\|\mathbf{s}_{n}-\mathbf{t}\right\|}{c}\right)} d t$

is the autocorrelation function of the transmitted waveform $\tilde{p}(t)$ that determines the range resolution of the radar system, $\eta_{n}(\mathbf{x})=\left[R\left(\mathbf{s}_{n}, \mathbf{x}, \mathbf{t}\right)-\left\|\mathbf{s}_{n}-\mathbf{t}\right\|\right] / c$ denotes the difference of time delay, and

$$
\kappa_{n}(\mathbf{x})=\eta\left(\mathbf{x}, \mathbf{s}_{n}\right) A\left(\omega_{0}, \mathbf{x}, \mathbf{s}_{n}\right)
$$

denotes the complex scattering coefficient. It should be noted that the scattering coefficients are dependent on the observation position $\mathbf{s}_{n}$. In the conventional SAR imaging system, it is reasonable to assume that the scattering coefficient of each scatterer is invariant during the entire period of radar beam illumination due to the narrow observation aperture. It is impractical, however, for this same assumption to be valid in the underlying wide-angle passive SAR system that is operated with a low carrier frequency. The scattering coefficients become even more distinct when a number of widely distributed receivers are used to acquire multiple, possibly disjoint, sub-apertures. Without loss of generality, we divide the entire observation aperture into $L$ smaller observation sub-apertures (groups). The scattering coefficients are assumed to be invariant within each sub-aperture, whereas they vary across different sub-apertures. As a result, there are $L$ groups of incoherent echo data of the scene. Let $D$ be the number of azimuth positions of the receiver within each group, and $L D=N$.

Now we discretize the sparse target scene into $M$ pixels. The sparse scene is represented by $q$ targets with a continuous structure, occupying a total number of $Q$ pixels in the entire $M$-pixel region, where $Q \ll M$, whereas the reflectivity of the other $M-Q$ pixels are assumed to be negligible. In this case, (5) becomes

$$
g\left(\tau, \mathbf{s}_{n}, \mathbf{t}\right)=\sum_{m=1}^{M} C_{0} \psi_{n}(\tau, m) \kappa_{m n}
$$


Denote $g\left(\tau, \mathbf{s}_{n}, \mathbf{t}\right) / C_{0}$ by $g_{n}(\tau)$ for simplicity. Then, by stacking the observations over all sampled delays, i.e., $\mathbf{g}_{n}=$ $\left[g_{n}\left(\tau_{1}\right), \ldots, g_{n}\left(\tau_{B}\right)\right]^{T}$, where $B$ is the number of range cells being observed, we obtain

$$
\mathbf{g}_{n}=\boldsymbol{\Psi}_{n} \boldsymbol{\kappa}_{n}+\varepsilon_{n},
$$

where $\boldsymbol{\Psi}_{n}=\left[\boldsymbol{\psi}_{n}^{T}\left(\tau_{1}\right), \cdots, \boldsymbol{\psi}_{M n}^{T}\left(\tau_{B}\right)\right]^{T}$, in which $\boldsymbol{\psi}_{n}(\tau)=$ $\left[\psi_{n}(\tau, 1), \cdots, \psi_{n}(\tau, M)\right]$, is the observation matrix corresponding to azimuth position $\mathbf{s}_{n}$ corresponding to all $M$ pixels of $\kappa_{m n}$ collected in $\boldsymbol{\kappa}_{n}=\left[\kappa_{1 n}, \ldots, \kappa_{M n}\right]^{T}$, and $\boldsymbol{\varepsilon}_{n}$ is a vector that accounts for the additive measurement noise. Note that $\boldsymbol{\kappa}_{n}$ is sparse in the sense that only $Q$ non-zero coefficients are dominant.

To account for the aspect-dependent scattering coefficients, as we described previously, we group the measured data corresponding to different aspect angles due to wide-angle and/or multi-angle observations into multiple tasks. In the multi-static case, each task corresponds to a bistatic observation. In the wide-angle case, the observations obtained in the $N$ azimuth positions are divided into $L$ tasks. i.e., $\left\{\mathbf{g}_{n}, n=1, \ldots, N\right\}$ is labeled as $\left\{\mathbf{g}_{1}^{(1)}, \ldots, \mathbf{g}_{D}^{(1)}, \ldots \mathbf{g}_{1}^{(l)}, \ldots, \mathbf{g}_{D}^{(l)}, \ldots, \mathbf{g}_{1}^{(L)}, \ldots, \mathbf{g}_{D}^{(L)}\right\}$. The observation matrix $\boldsymbol{\Psi}_{n}$ and sparse vector $\boldsymbol{\kappa}_{n}$ are similarly arranged. The scattering coefficients in $\boldsymbol{\kappa}_{n}$ are considered aspect-independent within the same task, and they vary independently for different tasks.

By stacking those echoes with respect to the observation aperture positions which belong to the same task (group), say, the $l$ th task, we have

$$
\mathbf{y}_{l}=\mathbf{\Phi}_{l} \mathbf{w}_{l}+\boldsymbol{\epsilon}_{l}, \quad l \in[1, \cdots, L],
$$

where

$$
\begin{aligned}
\mathbf{y}_{l} & \left.=\left[\mathbf{g}_{1}^{(l)^{T}}, \cdots, \mathbf{g}_{D}^{(l)}\right]^{T}\right]^{T}, \\
\mathbf{\Phi}_{l} & =\left[\mathbf{\Psi}_{1}^{(l)^{T}}, \cdots, \mathbf{\Psi}_{D}^{(l)}\right]^{T}, \\
\mathbf{w}_{l} & =\left[\boldsymbol{\kappa}_{1}^{(l)^{T}}, \cdots, \boldsymbol{\kappa}_{D}^{(l)^{T}}\right]^{T}, \\
\boldsymbol{\epsilon}_{l} & \left.=\left[\varepsilon_{1}^{(l)^{T}}, \cdots, \boldsymbol{\varepsilon}_{D}^{(l)}\right]^{T}\right]^{T} .
\end{aligned}
$$

It should be noted that the supports of the sparse targets are approximately identical across the $L$ groups (i.e., the non-zero entries of $\mathbf{w}_{l}$ lie in the same positions across different values of $l$ ), whereas their scattering coefficients are different.

Equation (12) is a typical discrete forward model of group sparse apertures for a passive imaging radar system. As such, the radar imaging problem in wide-angle and/or multi-angle passive SAR system can be regarded as the reconstruction of $L$-group sparse scattering coefficients.

\section{Proposed Structure-Aware Bayesian Compressive Sensing Algorithm}

In this section, we first introduce the spike-and-slab prior [51-53] in Section III-A. Based on the spike-and-slab prior platform, we then present in Section III-B the proposed structure-aware BCS algorithm using a novel logistic Gaussian kernel.

\section{A. Spike-and-slab prior for signal sparsity}

Consider a clustered sparse reconstruction problem with $L$ tasks, each consisting of $M$ entries. To encourage the group sparsity described in (12), we place a spike-and-slab prior to $\mathbf{w}_{l}$, i.e., [51-53]

$$
p\left(\mathbf{w}_{l} \mid \boldsymbol{\pi}, \boldsymbol{\beta}\right)=\prod_{i=1}^{M}\left[\left(1-\pi_{i}\right) \delta\left(w_{i l}\right)+\pi_{i} \mathcal{C N}\left(w_{i l} \mid 0, \beta_{l}^{-1}\right)\right],
$$

where $\pi_{i}$ is the prior probability of a non-zero element, i.e., a large weight $\pi_{i}$ corresponds to a high probability that the entry takes a non-zero value, whereas a small $\pi_{i}$ tends to generate a zero entry. In addition, $\beta_{l}$ is the precision (reciprocal of the variance) of the Gaussian distribution.

To facilitate the inference of (17) which is complicated by the involvement of the delta function, a simple reparameterization of the spike-and-slab prior is introduced in [5153]. This prior exploits a complex Gaussian random vector $\boldsymbol{\theta}_{l}=\left[\theta_{1 l}, \ldots, \theta_{M l}\right]^{T}$ with $p\left(\boldsymbol{\theta}_{l}\right)=\prod_{i=1}^{M} \mathcal{C N}\left(\theta_{i l} \mid 0, \beta_{l}^{-1}\right)$, $l=1, \ldots, L$ and a Bernoulli random vector $\mathbf{z}=\left[z_{1}, \ldots, z_{M}\right]^{T}$ with $p(\mathbf{z})=\prod_{i=1}^{M} \operatorname{Bern}\left(z_{i} \mid \pi_{i}\right)$, where $z_{i}=1$ corresponds to a non-zero entry in the $i$ th position. The product of these two latent vectors, $\boldsymbol{\theta}_{l} \circ \mathbf{z}$, forms a new random vector that follows the pdf in (17), i.e.,

$$
\mathbf{w}_{l}=\boldsymbol{\theta}_{l} \circ \mathbf{z} .
$$

In this expression, the group sparsity is characterized by the same $z_{i}$ for the $i$ th position of $\boldsymbol{\theta}_{l}$ across all $L$ tasks. On the other hand, scattering coefficients in the $i$ th block $\boldsymbol{\theta}_{i}$. $=\left[\theta_{i 1}, \cdots, \theta_{i L}\right]$ generally assume different values for each task. Considering the fact that $\boldsymbol{\theta}_{l}$ and $\mathbf{z}$ are not independent but rather strongly correlated, the following paired spike-and-slab prior

$$
p\left(\boldsymbol{\theta}_{l}, \mathbf{z}\right)=\prod_{i=1}^{M}\left[\mathcal{C N}\left(\theta_{i l} \mid 0, \beta_{l}^{-1}\right)\right]^{z_{i}} \pi_{i}^{z_{i}}\left(1-\pi_{i}\right)^{1-z_{i}}
$$

is introduced in [54] to enhance the sparse reconstruction performance.

To acquire the trackable posterior of $\beta_{l}$, we place a Gamma prior, which is conjugate to the Gaussian distribution, on $\beta_{l}$, i.e., $\beta_{l} \sim \operatorname{Gamma}\left(a_{l}, b_{l}\right), l \in[1, \cdots, L]$, where $a_{l}$ and $b_{l}$ are, respectively, the $l$ th element of hyper-parameter vectors $\mathbf{a}=\left[a_{1}, \cdots, a_{l}, \cdots, a_{L}\right]^{T}$ and $\mathbf{b}=\left[b_{1}, \cdots, b_{l}, \cdots, b_{L}\right]^{T}$. Similarly, we place a complex Gaussian prior on the additive noise as $\boldsymbol{\epsilon}_{l} \sim \mathcal{C N}\left(\boldsymbol{\epsilon}_{l} \mid \mathbf{0}, \alpha_{l}^{-1} \mathbf{I}_{N}\right)$, and a Gamma prior on $\alpha_{l}$ to acquire an analytical posterior distribution, i.e., $\alpha_{l} \sim \operatorname{Gamma}\left(c_{l}, d_{l}\right), l \in[1, \cdots, L]$, where $c_{l}$ and $d_{l}$ are respectively the $l$ th entries of the hyper-parameter vectors $\mathbf{c}=\left[c_{1}, \cdots, c_{l}, \cdots, c_{L}\right]^{T}$ and $\mathbf{d}=\left[d_{1}, \cdots, d_{l}, \cdots, d_{L}\right]^{T}$.

\section{B. Logistic Gaussian kernel model}

To encourage the continuous structure of target scatterers, a logistic Gaussian kernel model is proposed [46]. Unlike the methods described in $[39-42,53]$, which manually categorize a small number of predefined patterns and assign corresponding hyper-parameters for each pattern to encourage the clustered structure, the proposed algorithm achieves this objective 
through a kernel method that exploits the physical location of pixels within the region of interest. Therefore, the proposed algorithm has the capability to model the relationship among all pixels and acquire enhanced reconstruction performance.

In Section III-A, the variable $z_{i}$ follows a Bernoulli distribution with weight $\pi_{i}$. We employ a logistic function to express $\pi_{i}$ in term of $\gamma_{i}$ as

$$
\pi_{i}=\frac{1}{1+e^{-\rho \gamma_{i}}}
$$

where $\rho$ is a scale parameter. Denote $\gamma=\left[\gamma_{1}, \cdots, \gamma_{M}\right]^{T}$. To model the continuous structure of the pixels' support in the discretized target scene, a Gaussian kernel is introduced to $\gamma$ and is expressed as,

$$
\gamma \sim \mathcal{N}(0, \boldsymbol{\Sigma})
$$

in which the $(i, j)$ th element of $\boldsymbol{\Sigma}$ is given as

$$
\Sigma_{i j}=\exp \left(-\frac{\left\|\mathbf{x}_{i}-\mathbf{x}_{j}\right\|^{2}}{2 \sigma_{0}}\right)
$$

for $i, j \in(1, \cdots, M)$, where $\mathbf{x}_{i}$ and $\mathbf{x}_{j}$ are physical locations of the $i$ th and $j$ th pixels within the image, and $\sigma_{0}>0$ is a scale parameter.

Notice in Eq. (21) that the kernel matrix is real, symmetric, and, in the one-dimensional case, Toeplitz. All entries take values between 0 and 1 . The diagonal entries take the value of unity because $\left\|\mathbf{x}_{i}-\mathbf{x}_{j}\right\|^{2}=0$ for $i=j$, and the values decrease for off-diagonal elements, depending on the distance between $\mathbf{x}_{i}$ and $\mathbf{x}_{j}$. When all other pixels are involved in deciding the prior probability of a pixel under consideration, those pixels that are closer to the underlying pixel have stronger influence. As such, the use of the logistic model amounts to employing soft-thresholding weights rather than hard-thresholding ones as used in the existing methods [3942, 53].

It should be pointed out that $\sigma_{0}$ is an important parameter that determines the shape of Gaussian function and, thereby, the locality scale of the signal structure. When $\sigma_{0}$ approaches infinity, all entries in matrix $\boldsymbol{\Sigma}$ would approach 1 . It represents that the supports of all the pixels in the discretized target scene are highly correlated. When $\sigma_{0}$ approaches 0 , on the other hand, $\boldsymbol{\Sigma}$ is an identity matrix and the model will reduce to a typical sparse regression without consideration of the location dependence. Following the tradition, $\sigma_{0}$ is assigned to be an exponent of 2. In practice, the value of $\sigma_{0}$ can be learned from the measurement data by utilizing the maximum a posterior (MAP) or maximum likelihood (ML) estimation [55]. In the simulation and experimental studies, it is found that an empirical $\sigma_{0}$ is easily acquired. We also demonstrate the effect of the choice of $\sigma_{0}$ on the reconstruction performance.

\section{POSTERIOR INFERENCE}

In this section, we adopt a Gibbs sampler to perform Bayesian inference. For convenience, we define the collection of hyper-parameters as $\boldsymbol{\Xi} \triangleq\left\{\mathbf{a}, \mathbf{b}, \mathbf{c}, \mathbf{d}, \sigma_{0}\right\}$ and the collection of random variables as $\Theta \triangleq\left\{\left[\boldsymbol{\theta}_{l}\right]_{l=1}^{L}, \mathbf{z}, \boldsymbol{\pi}, \boldsymbol{\alpha}, \boldsymbol{\beta}, \rho, \boldsymbol{\gamma}\right\}$. We also denote $\mathbf{Y}=\left\{\mathbf{y}_{1}, \cdots, \mathbf{y}_{L}\right\}$ and $\boldsymbol{\Phi}=\left\{\boldsymbol{\Phi}_{1}, \cdots, \boldsymbol{\Phi}_{L}\right\}$.
According to the generative model described in Section III, we acquire the explicit form of the joint pdf as

$$
\begin{aligned}
p(\mathbf{Y}, \boldsymbol{\Phi}, \boldsymbol{\Theta} \mid \boldsymbol{\Xi}) & =\prod_{l=1}^{L} \mathcal{C N}\left(\mathbf{y}_{l} \mid \boldsymbol{\Phi}_{l}\left(\boldsymbol{\theta}_{l} \circ \mathbf{z}\right), \alpha_{l}^{-1} \mathbf{I}\right) \\
& \times \prod_{l=1}^{L} \prod_{i=1}^{M}\left[\mathcal{C N}\left(\theta_{i l} \mid 0, \beta_{l}^{-1}\right)\right]^{z_{i}} \pi_{i}^{z_{i}}\left(1-\pi_{i}\right)^{1-z_{i}} \\
& \times \prod_{i=1}^{M} \operatorname{Bern}\left[\frac{1}{1+e^{-\rho \gamma_{i}}}\right] \mathcal{N}(\gamma \mid 0, \boldsymbol{\Sigma}) \\
& \times \prod_{l=1}^{L} \operatorname{Gamma}\left(\beta_{l} \mid a_{l}, b_{l}\right) \operatorname{Gamma}\left(\alpha_{l} \mid c_{l}, d_{l}\right)
\end{aligned}
$$

In the following, we perform Gibbs sampling to obtain the posterior distributions for each latent variable based on the above joint pdf.

\section{A. Updating paired variables $\left\{\left[\boldsymbol{\theta}_{l}\right]_{l=1}^{L}, \mathbf{z}\right\}$}

Similar to [41], the paired MCMC sampler iteratively samples from the following conditional pdf

$p\left(z_{i}, \theta_{i l} \mid \boldsymbol{\theta}_{\backslash i l}, \mathbf{z}_{\backslash i}, \mathbf{y}_{l}\right)=p\left(\theta_{i l} \mid z_{i}, \boldsymbol{\theta}_{\backslash i l}, \mathbf{z}_{\backslash i}, \mathbf{y}_{l}\right) p\left(z_{i} \mid \boldsymbol{\theta}_{\backslash i l}, \mathbf{z}_{\backslash i}, \mathbf{y}_{l}\right)$,

where $\boldsymbol{\theta}_{\backslash i l}$ and $\mathbf{z}_{\backslash i}$ respectively denote $\boldsymbol{\theta}_{l}$ except the variable $\theta_{i l}$ and $\mathbf{z}$ except the variable $z_{i}$.

The probability $p\left(z_{i}=1 \mid \boldsymbol{\theta}_{\backslash i l}, \mathbf{z}_{\backslash i}, \mathbf{y}_{l}\right)$ is acquired analytically by utilizing the logistic function, expressed as,

$$
p\left(z_{i}=1 \mid \boldsymbol{\theta}_{\backslash i l}, \mathbf{z}_{\backslash i}, \mathbf{y}_{l}\right)=\frac{1}{1+e^{-u_{i}}},
$$

where $u_{i}$ is derived as

$$
\begin{aligned}
u_{i} & =\frac{1}{2} \sum_{l=1}^{L}\left(\log \beta_{l}+\log \sigma_{i l}+\sigma_{i l} \alpha_{l}^{2} \mathbf{y}_{\backslash i l}^{H} \boldsymbol{\phi}_{i l} \boldsymbol{\phi}_{i l}^{H} \mathbf{y}_{\backslash i l}\right)+\rho \gamma_{i}, \\
\sigma_{i l} & =\frac{1}{\alpha_{l} \boldsymbol{\phi}_{i l}^{H} \boldsymbol{\phi}_{i l}+\beta_{l}},
\end{aligned}
$$

with $\mathbf{y}_{\backslash i l}=\mathbf{y}_{l}-\sum_{k \neq i} \phi_{k l} z_{k} \theta_{k l}$, and $\phi_{i l}$ is the $i$ th column in the measurement matrix $\boldsymbol{\Phi}_{l}$. When $z_{i}=1$, the conditional distribution of $p\left(\theta_{i l} \mid z_{i}=1, \boldsymbol{\theta}_{\backslash i l}, \mathbf{z}_{\backslash i}, \mathbf{y}_{l}\right)$ can be expressed as,

$$
p\left(\theta_{i l} \mid z_{i}=1, \boldsymbol{\theta}_{\backslash i l}, \mathbf{z}_{\backslash i}, \mathbf{y}_{l}\right)=\mathcal{N}\left(\theta_{i l} \mid \sigma_{i l} \alpha_{l} \boldsymbol{\phi}_{i l}^{H} \mathbf{y}_{\backslash i l}, \sigma_{i l}\right) .
$$

For $z_{i}=0$, because the value of $\theta_{i l}$ does not affect the result of $w_{i l}$, we conveniently draw the value of variable $\theta_{i l}$ from its prior.

\section{B. Updating variables $\gamma$}

It is generally difficult to perform inference for variable $\gamma$, due to the analytically inconvenient form of the model's likelihood function [56]. Some strategies, such as analytic approximations, numerical integration, and Metropolis-Hastings, can be used to perform the approximation of complex pdf [55]. In the proposed model, however, we acquire an analytical posterior inference for $\gamma$ by following the auto-augmentation 
technique for the logistic model, which introduces a PG random variable vector. In [57], a fundamental integral identity is used as,

$$
\frac{\left(e^{x}\right)^{a_{0}}}{\left(1+e^{x}\right)^{b_{0}}}=2^{-b_{0}} e^{\left(a_{0}-\frac{b_{0}}{2}\right) x} \int_{0}^{\infty} e^{-q x^{2} / 2} f(q) d q,
$$

where $f(q)=\operatorname{PG}\left(q \mid b_{0}, 0\right)$. The function $\operatorname{PG}\left(q \mid c_{0}, d_{0}\right)$ can be expressed by,

$$
\begin{aligned}
\operatorname{PG}\left(q \mid c_{0}, d_{0}\right) & =\sum_{n=0}^{\infty}(-1)^{n} \frac{\Gamma\left(n+c_{0}\right) \Gamma\left(2 n+c_{0}\right)}{\Gamma(n+1) \sqrt{2 \pi q^{3}}} \\
& \times \frac{\cosh ^{c_{0}}\left(d_{0} / 2\right) 2^{c_{0}-1}}{\Gamma\left(c_{0}\right)} e^{-\frac{\left(2 n+c_{0}\right)^{2}}{8 q}-\frac{d_{0}^{2} q}{2}}
\end{aligned}
$$

where $\Gamma(\cdot)$ is a Gamma function. By introducing the latent PG variable $q$, the logistic model in Eq. (29) can be achieved by a hierarchical sampling structure, where the main parameter $x$ follows the Gaussian distribution with parameters in term of $q$, whereas $q$ in a lower layer follows the PG distribution.

Using the integral identity in Eq. (29), the weight in the logistic model can be expressed by

$$
\frac{\left(e^{\rho \gamma_{i}}\right)^{z_{i}}}{\left(1+e^{\rho \gamma_{i}}\right) z_{i}}=2^{-z_{i}} e^{\frac{z_{i}}{2} \rho \gamma_{i}} \int_{0}^{\infty} e^{\frac{-q_{i} \rho^{2} \gamma_{i}^{2}}{2}} p\left(q_{i}\right) d q_{i} .
$$

In a similar manner, we acquire,

$$
\frac{\left(e^{-\rho \gamma_{i}}\right)^{1-z_{i}}}{\left(1+e^{-\rho \gamma_{i}}\right)^{1-z_{i}}}=2^{\left(z_{i}-1\right)} e^{\frac{z_{i}-1}{2} \rho \gamma_{i}} \int_{0}^{\infty} e^{\frac{-q_{i} \rho^{2} \gamma_{i}^{2}}{2}} p\left(q_{i}\right) d q_{i} \text {. }
$$

According to Eq. (31) and Eq. (32), we acquire the analytical posterior distribution of $\mathbf{q}$ as

$$
\begin{aligned}
p(\gamma \mid \mathbf{q}, \mathbf{z}, \boldsymbol{\Sigma}) & \propto \mathcal{N}(\boldsymbol{\gamma} \mid \boldsymbol{\nu}, \boldsymbol{\Lambda}) \mathcal{N}(\mathbf{x}, \mathbf{0}, \boldsymbol{\Sigma}) \\
& =\mathcal{N}(\boldsymbol{\gamma} \mid \boldsymbol{\mu}, \boldsymbol{\Gamma}),
\end{aligned}
$$

with

$$
\begin{aligned}
& \boldsymbol{\mu}=\boldsymbol{\Gamma}^{-1} \boldsymbol{\Lambda} \boldsymbol{\nu}, \\
& \boldsymbol{\Gamma}=\left[\boldsymbol{\Lambda}+\boldsymbol{\Sigma}^{-1}\right]^{-1},
\end{aligned}
$$

where $\boldsymbol{\Lambda}=\operatorname{diag}\left(2 q_{1} \rho^{2}, \cdots, 2 q_{1} \rho^{2}\right)$ and $\boldsymbol{\nu}=$ $\left[\left(z_{1}-1 / 2\right) /\left(2 q_{1} \rho\right), \cdots,\left(z_{M}-1 / 2\right) /\left(2 q_{M} \rho\right)\right]^{T}$. The derivation of (33) is provided in Appendix A. In addition, the augmented variable $q_{i}$ is updated as,

$$
p\left(q_{i} \mid \gamma_{i}\right)=\operatorname{PG}\left(q_{i} \mid 1, \gamma_{i}\right) \text {. }
$$

\section{Updating the scale parameter $\rho$}

It is difficult to perform inference for the scale parameter $\rho$, due to the inconvenient form. The following ML estimator is used,

$$
\begin{aligned}
\rho & =\arg \max _{\rho}[\log p(\mathbf{y}, \boldsymbol{\Theta})] \\
& =\arg \max _{\rho} \sum_{i=1}^{M}\left[\log \operatorname{Bern}\left(\frac{1}{1+e^{-\rho \gamma_{i}}}\right)\right] .
\end{aligned}
$$

We take gradient descent for Eq. (37) and acquire the updated expression of $\rho$ as,

$$
\rho=-\sum_{i=1}^{M} \frac{1}{\gamma_{i}} \log \frac{1-\mathbb{E}\left(z_{i}\right)}{\mathbb{E}\left(z_{i}\right)}
$$

\section{Updating signal precision $\beta_{l}$}

By utilizing the conjugate property of the Gaussian and Gamma distributions, we analytically acquire the posterior distribution of the precision variable $\beta_{l}$ as

$$
p\left(\beta_{l} \mid a_{l}, b_{l}, \boldsymbol{\theta}\right)=\operatorname{Gamma}\left(\tilde{a}_{l}, \tilde{b}_{l}\right),
$$

where $\tilde{a}_{l}=a_{l}+(M / 2)$ and $\tilde{b}_{l}=b_{l}+(1 / 2) \sum_{i} \theta_{i l}^{2}$ for $l \in$ $[1, \cdots, L]$.

\section{E. Updating noise precision $\alpha_{l}$}

In a similar manner as $\beta_{l}$, we also obtain the posterior distribution of noise precision $\alpha_{l}$ as

$$
p\left(\alpha_{l} \mid c_{l}, d_{l}, \mathbf{y}_{l}, \boldsymbol{\Phi}_{l}, \boldsymbol{\theta}_{l}, \mathbf{z}\right)=\operatorname{Gamma}\left(\tilde{c}_{l}, \tilde{d}_{l}\right),
$$

where $\tilde{c}_{l}=c_{l}+(B D / 2)$ and $\tilde{d}_{l}=d_{l}+\left(\left\|\mathbf{y}_{l}-\boldsymbol{\Phi}_{l}\left(\boldsymbol{\theta}_{l} \circ \mathbf{z}\right)\right\|^{2} / 2\right)$ for $l \in[1, \cdots, L]$.

\section{F. Computation Complexity}

The proposed algorithm is summarized in Table I. Since the inference of model parameters is implemented by the MCMC sampler, which inherently requires sequential sampling, it is straightforward to show from the procedure of the proposed algorithm, depicted in Table I, that the computational cost of the proposed algorithm is mainly due to the matrix inversion in Eq. (35). While it involves two $M \times M$ matrix inversions, this can be simplified by using the matrix inversion lemma, yielding

$$
\boldsymbol{\Gamma}=\boldsymbol{\Lambda}^{-1}\left(\boldsymbol{\Lambda}^{-1}+\boldsymbol{\Sigma}\right)^{-1} \boldsymbol{\Sigma} .
$$

The above expression involves a single matrix inversion. The inversion of an $M \times M$ matrix generally requires $\mathcal{O}\left(M^{3}\right)$ operations, which is the main computation cost in the proposed method. Note that, for one-dimensional problems, the computation complexity of the above matrix inversion would reduce to $\mathcal{O}\left(M^{2}\right)$ operations because $\boldsymbol{\Sigma}$, and subsequently $\left(\boldsymbol{\Lambda}^{-1}+\boldsymbol{\Sigma}\right)$, is Toeplitz [58, 59]. On the other hand, compared to those Bayesian based greedy algorithms [24, 28], the proposed method based on the Gibbs sampling scheme requires more time due to going through all elements in each MCMC iteration. In general, the proposed method has acquired the improved reconstruction performance, while it has a relatively high computation burden.

\section{Simulation And ExPerimental Results}

In the following simulations, two metrics are used to evaluate the imaging performance. The first one is the normalized mean square error (NMSE) $\left\|\hat{\mathbf{w}}-\mathbf{w}_{\text {gen }}\right\|_{2}^{2} /\left\|\mathbf{w}_{\text {gen }}\right\|_{2}^{2}$, where $\hat{\mathbf{w}}$ is the estimate of the true signal vector $\mathbf{w}_{\text {gen }}$. We consider that an exact reconstruction is achieved when NMSE $<10^{-5}$. The second metric is the earth mover's distance (EMD), which is based on the minimum cost that must be paid to transform one distribution to the other. Let $w_{i}$ and $\hat{w}_{k}$, respectively, be the $i$ th element of $\mathbf{w}_{\text {gen }}$ and the $k$ th element of $\hat{\mathbf{w}}$. Then, the EMD metric is defined as $\operatorname{EMD}\left(\mathbf{w}_{\text {gen }}, \hat{\mathbf{w}}\right)=\left(\sum_{i} \sum_{k} d_{i k} f_{i k}\right) /\left(\sum_{i} \sum_{k} f_{i k}\right)$, where $d_{i k}$ is the ground distance between $w_{i}$ and $\hat{w}_{k}$, and $f_{i k}$ is the 
Table I. Proposed BCS algorithm

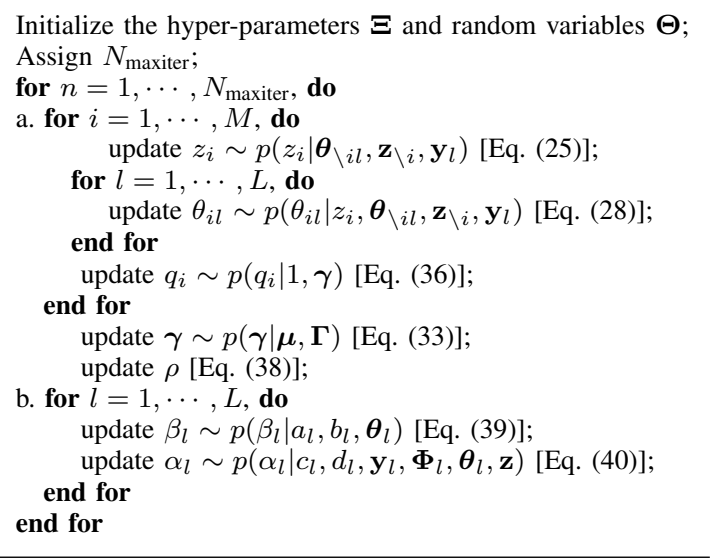

flow between $w_{i}$ and $\hat{w}_{k}$ [60]. The EMD metric allows us to evaluate the similarity between the true and the estimated results, with the displaced distance of the pixels taken into account. The maximum number of iterations in the Gibbs sampling is 600 , and the sample with the maximum marginal likelihood in the last 100 samples is chosen as the estimate of the scattering coefficients.

\section{A. High-resolution imaging in the wide-angle SAR system}

We first demonstrate the resolution enhancement in a wideangle observations system using the proposed approach. The DVB-T signals use OFDM modulation with 8192 subcarriers. The OFDM symbol has a duration of $1024 \mu \mathrm{s}$ for active subcarriers [61]. A center carrier frequency of $850 \mathrm{MHz}$ and a bandwidth of $7.8 \mathrm{MHz}$ are used, and the received data is sampled at a sampling rate of $10 \mathrm{MHz}$ in the simulations. The speed of the moving receiver is $150 \mathrm{~m} / \mathrm{s}$ along the $\mathrm{y}$ axis. The conventional range and azimuth resolutions are about $20 \mathrm{~m}$ and $2 \mathrm{~m}$ in each sub-apertures, respectively. The resolutions, especially that in range, are greatly improved as a result of sparse reconstruction. As shown in Fig. 2(a), a $15^{\circ}$ observation aperture is considered and is divided into 3 sub-apertures, where the resulting observation angle in each sub-aperture is $5^{\circ} .64$ synthetic aperture positions are acquired by uniformly dividing each sub-aperture width. The complex scattering coefficients of the sparse targets are assumed to vary with the sub-aperture, but remain unchanged within the same sub-aperture. Unless otherwise specified, the raw-data input SNR is set to $-30 \mathrm{~dB}$, and $\sigma_{0}$ is chosen to be 16 .

The sparse scene being considered, as depicted in Fig. 3(a), consists of $32 \times 32$ pixels. With the consideration of the sparsity measure [62] that most signals can be recovered by the $l_{1}$-norm minimization, we consider two continuous targets $(q=2)$, whose shapes are cross and square, respectively. Each target consists of 9 non-zero pixels, yielding a combined sparsity of $Q=18$. The inter-pixel spacing is $1 \mathrm{~m}$ in both range and azimuth directions.

According to the conventional SAR imaging principle, a high image resolution is achieved by exploiting a wide coherent synthetic aperture. However, we cannot coherently accumulate the data acquired over the entire aperture width

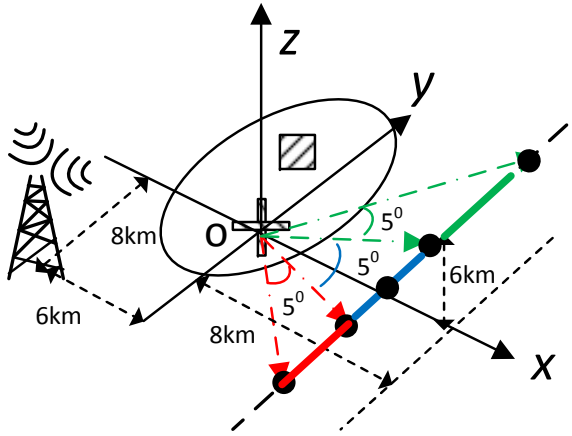

(a)

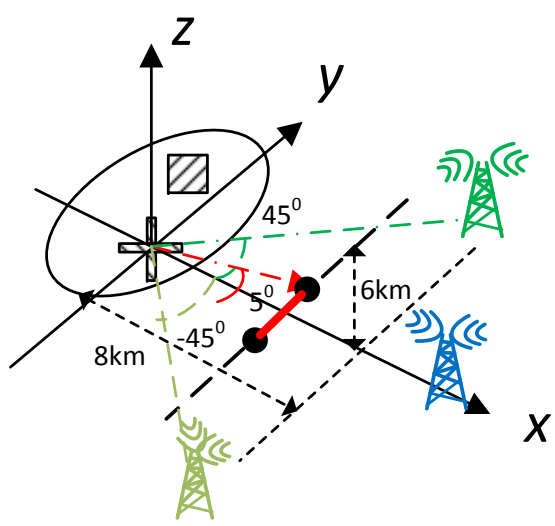

(b)

Fig. 2. (a) Geometry of passive wide-angle SAR system, and (b) Geometry of passive multi-angle SAR system.

because the target scattering coefficients vary with the aspect angle. As such, without considering the group sparsity, we have to form individual images separately for the three subapertures. The resulting images by individually applying the proposed method to each of the three sub-apertures are respectively shown in Figs. 3(d)-3(f). The result of incoherent fusion of these three images is shown in Fig. 3(c). It is clear from the fused image that the targets within one conventional range cell $(20 \mathrm{~m})$ are resolved, but the resulting image shows missing target entries as well as spurious target entries.

By considering the group sparsity between sub-aperture images and the underlying continuous structures of the targets, the reconstructed result exploiting the proposed method with $L=3$ sub-apertures is depicted in Fig. 3(b) in terms of the Frobenius norm of the complex scattering coefficients across the three sub-apertures. It is evident that the performance in Fig. 3(b) is superior to that in Fig. 3(c). The proposed algorithm with joint learning clearly recovers the sparse targets where the range and azimuth resolutions of the targets are as high as $1 \mathrm{~m}$. As discussed earlier, the consideration of group sparsity across the three sub-apertures allows the exploitation of the information observed from other sub-apertures to achieve improved performance. As such, the reconstructed result of the joint learning of 3 groups, as shown in Fig. 3(b), outperforms any single learning model depicted in Figs. 3(d)3(f), as well as the fused results depicted in Fig. 3(c).

Next, we compare the performance of the proposed method 


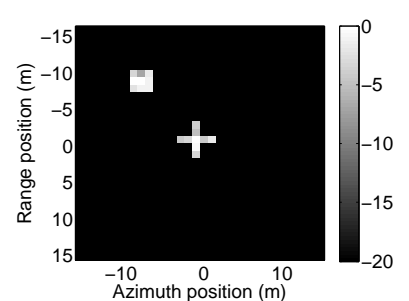

(a)

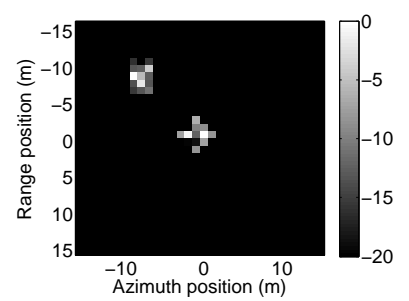

(c)

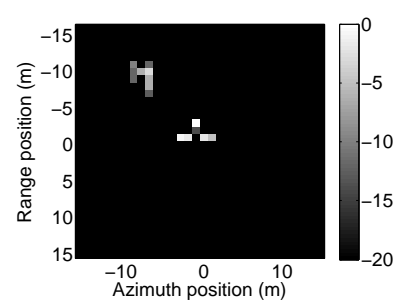

(e)

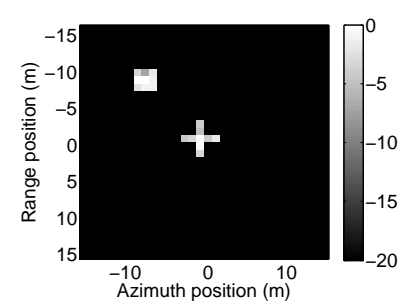

(b)

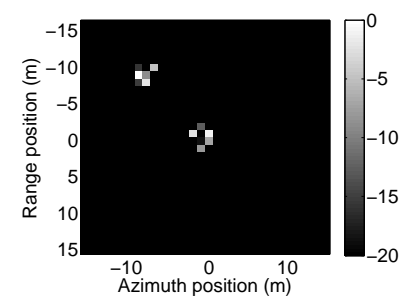

(d)

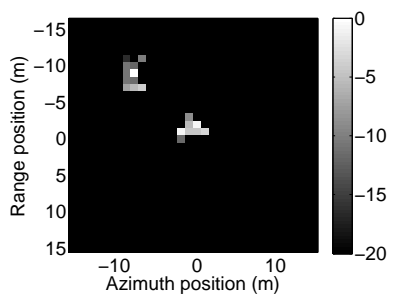

(f)
Fig. 3. (a) Normalized magnitude of the scattering coefficients of the original scene. (b) Reconstructed result acquired by incoherently fusing images individually obtained from the three sub-aperture groups ( $L=3$ ) using the proposed method. (c) Fused image based on three sub-images, respectively depicted in Figs. 3(d)-3(f). (d) Reconstructed result acquired by the proposed method using the first sub-aperture data. (e) Reconstructed result acquired by the proposed method using the second sub-aperture data. (f) Reconstructed result acquired by the proposed method using the third sub-aperture data.

to selected existing $\mathrm{CS}$ techniques in the sparse Bayesian learning framework, including the clustered BCS in [41], mt-CS [27] and conventional FBP. As shown in Fig. 4(a), the FBP fails to achieve a desirable resolution, yielding very poor image quality, because the FBP imaging algorithm does not have high-solution imaging capability and all the targets in the same range resolution cell cannot be resolved. The mt-CS algorithm, as one of sparse Bayesian learning methods, achieves a better performance than that in the FBP algorithm. As shown in Fig. 4(c), the clustered BCS, by exploiting the target structure via manually assigned patterns, improves the imaging performance as it effectively preserves the underlying continuous structure and suppresses the isolated noise components. However, the clustered BCS algorithm, which uses assigned patterns in the first-order neighboring region, is inferior when compared to the proposed method, which takes correlation between all scatterers into account.

Finally, we compare the computational complexity among these aforementioned algorithms in terms of the computer running time using Matlab 2010a with a Dell S2340L computer (Intel Core i7-4790 CPU and 16 GB RAM). Fig. 5(a) shows the running time with respect to the number of image pixels corresponding to scene sizes of $16 \times 16,32 \times 32,64 \times 64$,

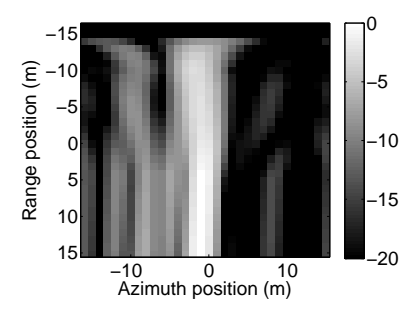

(a)

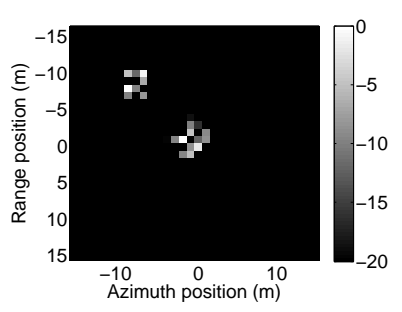

(b)

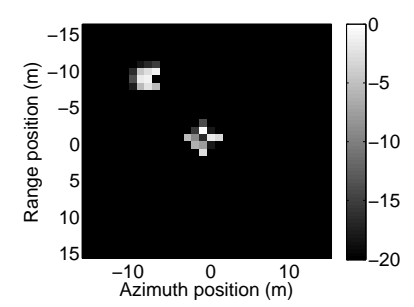

(c)

Fig. 4. (a) Reconstructed result based on FBP. (b) Reconstructed result based on mt-CS. (c) Reconstructed result based on clustered BCS.

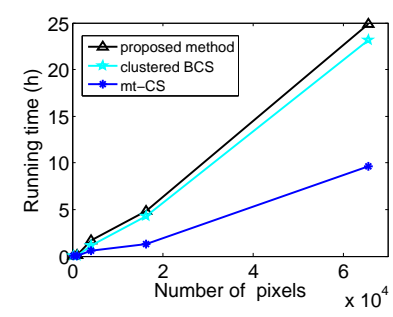

(a)

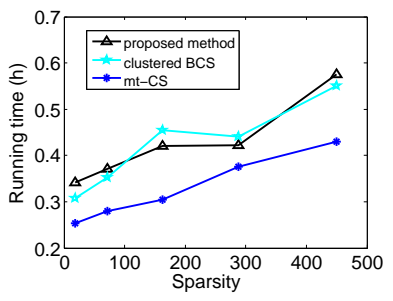

(b)

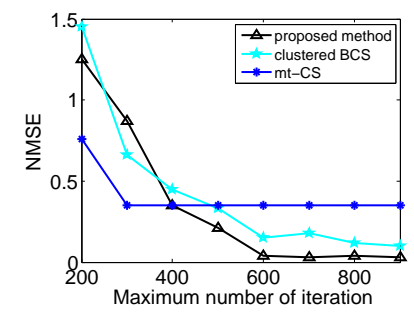

(c)

Fig. 5. (a) Running time versus scene size. (b) Running time versus sparsity (number of non-zero entries). (c) NMSE versus the maximum number of iteration.

$128 \times 128$, and $256 \times 256$. It is expected that the running time of all the algorithms increases with the number of pixels. Among the three methods, the complexity of the greedy based mt-CS algorithm is much smaller. In Fig. 5(b), we show the running time with respect to the sparsity for a scene of size $32 \times 32$. It is observed that the running time slowly increases with the number of non-zero entries, which are 18, 72, 162, 288, and 450, respectively. To further compare the performance of these algorithms, we show in Fig. 5(c) the output NMSE with respect to the maximum number of iterations for a $32 \times 32$ scene with sparsity 18 . It is clear that the proposed method acquires the lowest NMSE of less than 0.1 when the maximum number of iterations is larger than 600 . The mt-CS algorithm converges after 300 iterations, but the output NMSE remains a high level of 0.35 . 


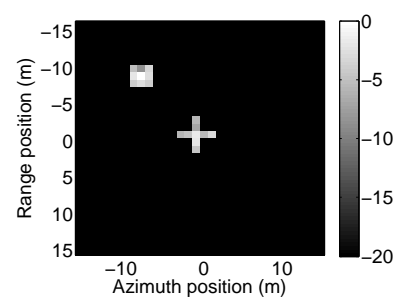

(a)

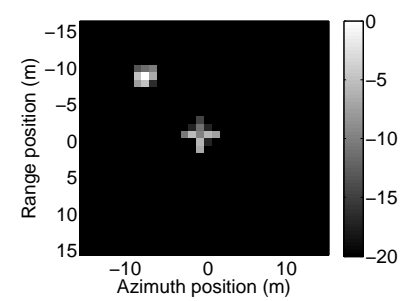

(b)

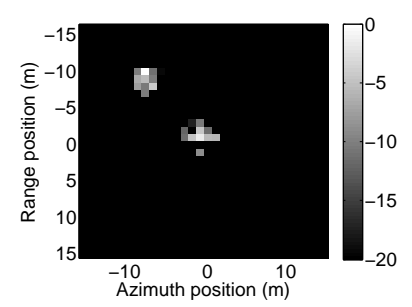

(c)

Fig. 6. Reconstruction results of the multi-angle SAR system. (a) proposed method, (b) clustered BCS, and (c) mt-CS.

\section{B. High-resolution imaging in the multi-angle SAR system}

An attractive feature of the passive radar system is its capability to exploit multiple spatially separated illuminators available in the scene to achieve a multi-static SAR configuration. Such a capability can be further enhanced when multiple distributed receivers are used. In this subsection, we consider a multiple-transmitter single-receiver model as an example of such a multi-angle SAR system. Considering the fact that different carrier frequencies are used in transmitted stations, we are able to separate these transmitted waveforms in the frequency spectrum. The simulation scene is illustrated in Fig. 2(b), which is similar to the wide-angle scenario considered earlier, except that we are now using three illuminators. The azimuth angle width of the receiver corresponding to each illuminator is $5^{\circ}$. The three illuminators are located 10 $\mathrm{km}$ away from the scene center, and their respective aspect angles are $-45^{\circ}, 0^{\circ}$, and $45^{\circ}$. These illuminators emit their individual DVB-T waveforms to the scene with different carrier frequencies. The complex scattering coefficients are assumed to be invariant with the $5^{\circ}$ angular span for each bistatic pair, but vary with different illuminators due to the distinct aspect angles.

The imaging results of the proposed method are compared with those obtained from the clustered BCS, mt-CS in Fig. 6. It is evident that the proposed algorithm accurately recovers all continuous targets with $\mathrm{NMSE}<0.1$, as shown in Fig. 6(a), and outperforms the other algorithms being compared. The clustered BCS method showing in Fig. 6(b) acquires a moderate reconstruction result with $\mathrm{NMSE}=0.24$. The mt-CS algorithm, as shown in Figs. 6(c), generally recovers inaccurate target profiles together with isolated noise components.

\section{Minimum grid interval analysis}

In the above examples, the proposed technique recovers high-fidelity target images with a 1-m resolution in both range and azimuth dimensions. In this subsection, we examine the minimum grid interval, which is considered as the passive radar imaging resolution, from the perspective of measurement matrix coherence. By the term minimum grid interval, we imply that exact sparse scene reconstruction is achieved when the entire scene, depicted in Fig. 3(a), is proportionally scaled, and the same grid interval is used for both range and azimuth dimensions.

For conventional CS algorithms, an exact reconstruction requires the measurement matrix $\boldsymbol{\Phi}^{(l)}$ for the $l$ th task, $l \in$ $[1, \cdots, L]$ to satisfy the restricted isometry property (RIP) condition which, in turn, implies the columns extracted from $\boldsymbol{\Phi}^{(l)}$ to be approximately orthogonal or at least to have a low coherence. However, this situation does not hold in the underlying high-resolution passive imaging system. The mutual coherence of the measurement matrix $\boldsymbol{\Phi}^{(l)}$ is defined as

$$
\rho^{(l)}=\rho\left(\boldsymbol{\Phi}^{(l)}\right)=\max _{m \neq m^{\prime}}\left|\rho_{m m^{\prime}}^{(l)}\right|
$$

where

$$
\rho_{m m^{\prime}}^{(l)}=\frac{\left(\phi_{m}^{(l)}\right)^{H} \boldsymbol{\phi}_{m^{\prime}}^{(l)}}{\left\|\boldsymbol{\phi}_{m}^{(l)}\right\|\left\|\boldsymbol{\phi}_{m^{\prime}}^{(l)}\right\|}
$$

and $\phi_{m}^{(l)}$ denotes the $m$ th column of $\boldsymbol{\Phi}^{(l)}$.

In the passive SAR system, $\rho_{m m^{\prime}}^{(l)}$ can be expressed as

$$
\rho_{m m^{\prime}}^{(l)}=\frac{1}{D} \sum_{i=1}^{D} \exp \left[j \omega_{0} \frac{R\left(\mathbf{s}_{i}, \mathbf{x}_{m}\right)-R\left(\mathbf{s}_{i}, \mathbf{x}_{m^{\prime}}\right)}{c}\right] .
$$

Using the triangle inequality, we obtain

$$
\rho_{m m^{\prime}}^{(l)} \leq \exp \left[j \frac{\omega_{0}}{c}\left\|\mathbf{x}_{m}-\mathbf{x}_{m^{\prime}}\right\|\right] .
$$

It is clear from this expression that both the grid interval and the carrier frequency affect the coherence of $\boldsymbol{\Phi}^{(l)}$. That is, a smaller distance between two adjacent grid pixels and a lower carrier frequency correspond to a higher coherence of $\boldsymbol{\Phi}^{(l)}$. This phenomenon is prominent in passive radar systems because their operating frequencies are generally low.

Consider two neighboring grids in azimuth, $\rho_{m m^{\prime}}^{(l)}$ can be written as [63],

$$
\rho_{m m^{\prime}}^{(l)} \leq \operatorname{sinc}\left(\frac{2 \omega_{0}}{c} \Delta X_{a} \tan \frac{\theta_{\mathrm{eff}}}{2}\right),
$$

where $\operatorname{sinc}(x)=\sin (x) / x, \Delta X_{a}$ is the azimuth spacing between two neighboring grid pixels, and $\theta_{\text {eff }}$ is the effective observation aperture width. Because the envelop of $\operatorname{sinc}(x)$ decreases with $x$ for $x>0$, reduction in the grid interval, carrier frequency, and the observation aperture will, in general, result in increased coherence and, subsequently, degraded sparse signal reconstruction performance.

Based on this discussion, we show in Fig. 7 the relationship between the observation aperture width and the minimum resolvable grid interval for the different algorithms being compared. It is evident that the proposed algorithm achieves the smallest resolvable grid interval, which is followed by the clustered BCS and the mt-CS. This means that the proposed algorithm offers a better sparse signal reconstruction capability. In other words, the proposed algorithm can reconstruct SAR images with a higher resolution. 


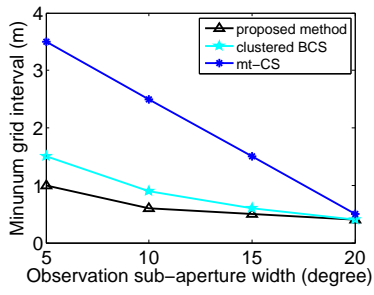

Fig. 7. Minimum grid interval varying with sub-aperture width.

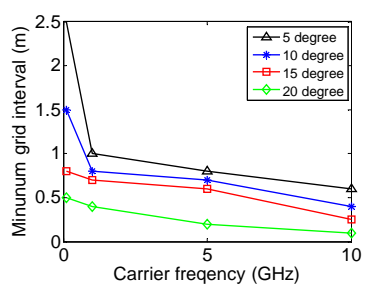

(a)

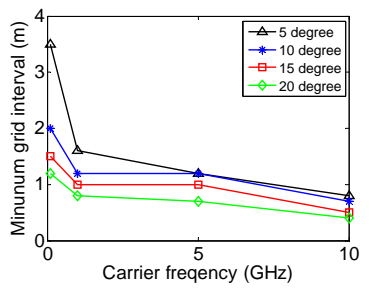

(b)

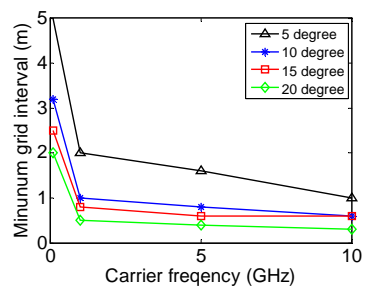

(c)

Fig. 8. Minimum grid interval with respect to carrier frequency based on different methods. (a) Proposed method, (b) clustered BCS, and (c) mt-CS.

Fig. 8 shows the relationship between the minimum grid interval and the carrier frequency for different sub-aperture widths. It is clear that the proposed algorithm achieves the smallest grid interval, i.e., the highest imaging resolution.

\section{Performance versus the number of azimuth samples}

In the simulations above, a $5^{\circ}$-width sub-aperture is uniformly divided into 64 aperture positions. In the following, we analyze the reconstruction performance with respect to the number of azimuth sample positions. To enable the comparison, we use a coarse grid interval of $20 \mathrm{~m}$ so that all the algorithms are able to reconstruct the sparse signals and their performance can be compared. All parameters are kept the same as those used in Section V-A and noiseless data observations are assumed. It is observed in Fig. 9(a) that the target scene can be exactly recovered by both the proposed algorithm and the clustered BCS when the number of azimuth samples is larger than 20. The number required to achieve exact recovery is 30 for the mt-CS. The superiority of the proposed technique is also confirmed in Fig. 9(b) that depicts the EMD versus the number of azimuth samples being used.

\section{E. Performance versus the support similarity}

All simulations above are performed under the assumption that the support of the sparse targets is identical across all

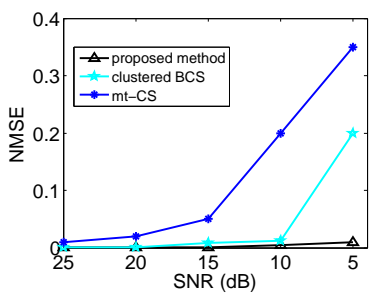

(a)

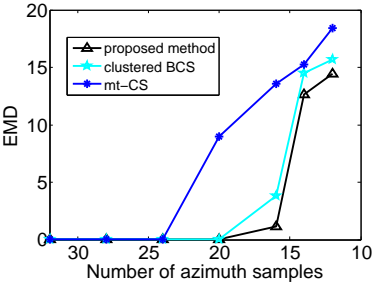

(b)
Fig. 9. (a) NMSE versus the number of azimuth samples, and (b) EMD versus the number of azimuth samples.

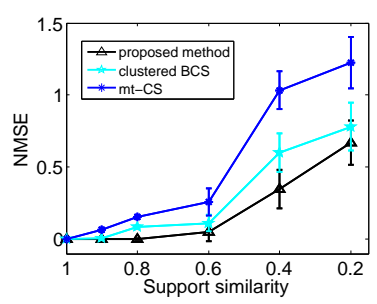

(a)

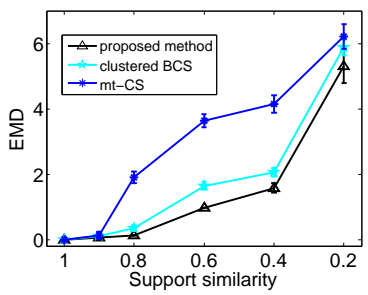

(b)
Fig. 10. (a) NMSE versus support similarity, and (b) EMD versus support similarity.

observation angles. In practice, the observed support of the sparse targets may vary with the observation angle. In this case, the proposed algorithm is still able to recover the sparse targets as long as the correlation of the sparse target supports for different observation angles is moderately high. Fig. 10(a) shows the relationship between the reconstruction NMSE performance and the support similarity, which is defined as the ratio between the number of common non-zero positions across different groups and the total number of nonzero positions. It is clear that the NMSE obtained from the proposed algorithm is very low as long as the support similarity is higher than $60 \%$. Similar results are observed in Fig. 10(b) that depicts the EMD performance as a function of the support similarity.

\section{F. Selection of the scale parameter $\sigma_{0}$}

To examine the effect of the scale parameter $\sigma_{0}$ in the Gaussian kernel on the reconstruction performance, we analyze the NMSE and the EMD versus $\sigma_{0}$ and provide a guidance for the selection of $\sigma_{0}$. The simulation parameters and grid interval follow those in Section V-D. Following the common practice, we take the value of $\sigma_{0}$ to be a factor of 2 between $2^{-2}$ and $2^{8}$. Figs. 11(a) and 11(b) respectively show the NMSE and EMD, averaged over 100 repeated trials, with respect to $\sigma_{0}$. It is observed that, when the number of azimuth samples is sufficiently high (the $D=32$ case in both figures), the target scene can be reconstructed very well, irrespective of the value of $\sigma_{0}$. On the other hand, a small number of azimuth samples (the $D=16$ case) yields high reconstruction errors. In this case, increasing $\sigma_{0}$ improve both NMSE and EMD, but the impact is rather limited. It is interesting to observe that, when a moderate number of azimuth samples $(D=20)$ is used, the value of $\sigma_{0}$ plays a much more significant role, underscoring the importance of effectively incorporating the 


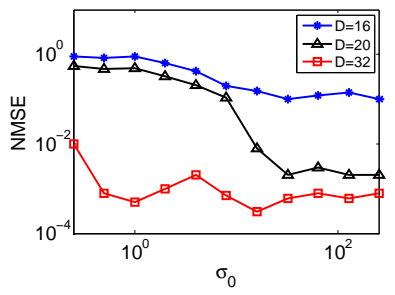

(a)

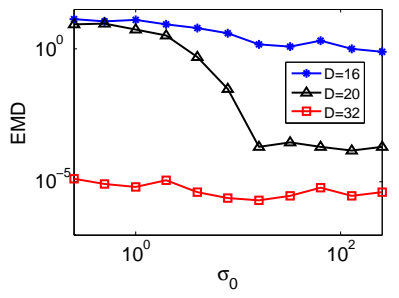

(b)
Fig. 11. Performance results versus the scale parameter $\sigma_{0}$ (a) NMSE, and (b) EMD.

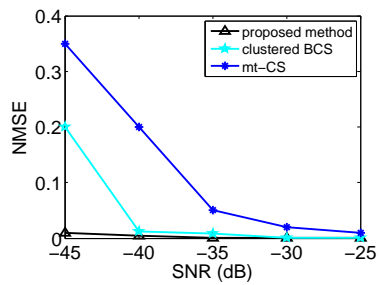

(a)

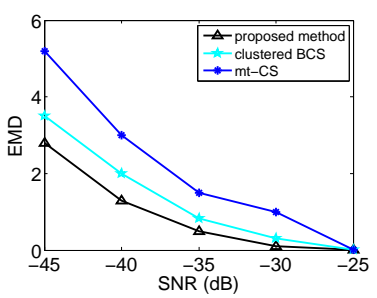

(b)
Fig. 12. (a) NMSE versus SNR, and (b) EMD versus SNR.

clustered structures. More specifically, both NMSE and EMD are sharply reduced when $\sigma_{0}$ is larger than $2^{4}$ in this case. In considering the size of the image, a value of $\sigma_{0}$ above $2^{8}$ is discouraged so as to avoid the singularity issue when performing matrix inversion in Eq. (35).

\section{G. Performance in noisy environments}

We now compare the performance of proposed method with the clustered BCS and the mt-CS, when the received signals are contaminated by different levels of noise. Complex Gaussian white noise is added to the observed signals so that the SNR varies from $-45 \mathrm{~dB}$ to $-25 \mathrm{~dB}$.

As seen in Fig. 12, both methods with the exploitation of the continuous structures, i.e., the clustered BCS and the proposed algorithm, exhibit significant performance gains against the additive noise as compared to the mt-CS algorithms.

\section{H. Experiment on ship dataset}

In this section, the multi-angle synthetic datasets based on the TerraSAR-X SAR oil tanker imagery with scene size of $64 \times 64$, as shown in Fig. 13(a), are used to validate the proposed algorithm [64, 65]. The range and azimuth resolution of the original SAR image is $1.5 \mathrm{~m} \times 2 \mathrm{~m}$. It is observed that the oil tanker ship body has considerately strong reflectivities due to the metallic material and strong corner reflections, compared to the weak reflections from sea clutters. Therefore, it is reasonable to consider the ship target as sparse within the image. In the experiment, two transmitters, respectively located at $-45^{\circ}$ and $0^{\circ}$, and one moving receiver are considered. We generate another synthetic observation dataset by randomly altering the phase and adding random perturbation on the original SAR imagery. Other radar system parameters follow those in Section V-B. To

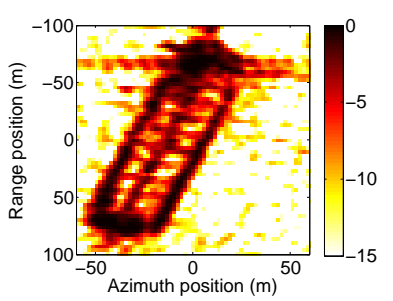

(a)

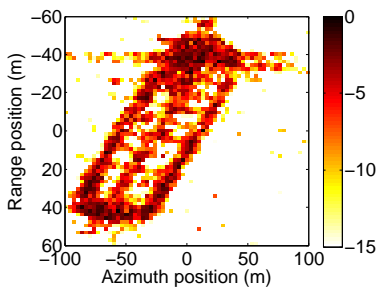

(c)

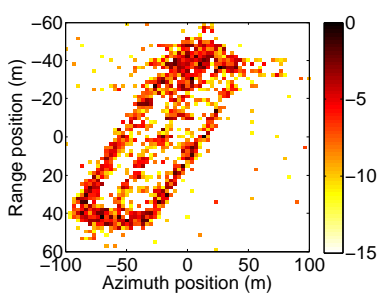

(b)

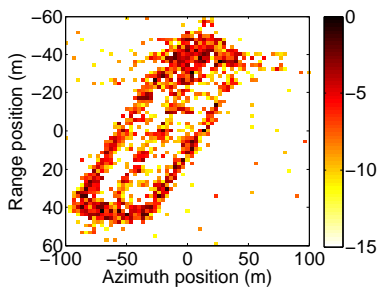

(d)

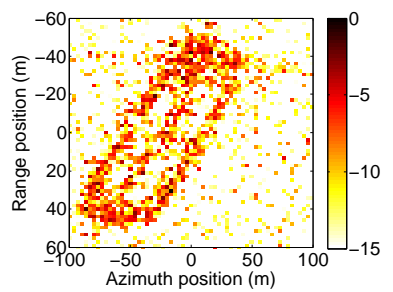

(e)

Fig. 13. Reconstructed results based on the oil tanker ship SAR image. (a) Original oil tanker ship SAR image. (b) Reconstructed result based on FBP with full dataset. (c) Reconstructed result based on the proposed method. (d) Reconstructed result based on clustered BCS. (e) Reconstructed result based on mt-CS.

quantitatively evaluate the quality of the recovered images, the image correlation coefficient, defined as

$$
\operatorname{Cor}\left(\mathbf{W}_{r}, \mathbf{W}_{e}\right)=\frac{\left\|\operatorname{vec}\left(\mathbf{W}_{r}\right) \circ \operatorname{vec}\left(\mathbf{W}_{e}\right)\right\|}{\left\|\operatorname{vec}\left(\mathbf{W}_{r}\right)\right\| \cdot\left\|\operatorname{vec}\left(\mathbf{W}_{e}\right)\right\|},
$$

is used as a as the metric, where $\mathbf{W}_{r}$ denotes the reference image, as shown in Fig. 13(a), and $\mathbf{W}_{e}$ denotes the reconstructed image based on the different CS techniques.

Fig. 13(b) shows the result based on the FBP with the full dataset. It is observed that the reconstructed image has very coarse range resolution. To demonstrate the performance of the CS technique, only 64 azimuth measurements are used in the sequel. As seen in Figs. 13(c)-13(e), all methods can properly recover most of the strong scatterers. However, compared to those obtained by mt-CS in Fig. 13(e) and gLasso in Fig. 13(e), the reconstructed images obtained from the proposed algorithm and the clustered BCS, which exploit the target structure, are much more concentrated. Since the correlation between all scatterers within the region of interest is exploited, the proposed method generally has an improved reconstruction performance and the resulting image correlation coefficient is 0.83 . For comparison, the image correlation coefficient is 0.79 for the clustered BCS, 0.71 for the mt-CS method.

\section{CONCLUSION}

Passive synthetic aperture radar (SAR) systems suffer from low range resolution due to their low carrier frequency and 
narrow signal bandwidth. A novel high-resolution imaging technique based on structured Bayesian compressive sensing (BCS) is proposed to achieve improved scene reconstruction through exploitation of the underlying target scene structure and group sparsity induced by wide-angle and/or multi-angle passive radar systems. The proposed structured BCS algorithm has the ability to acquire high-resolution images in wide-angle and/or multi-angle observations, where the scattering coefficients are assumed to change with the observation angles. The proposed technique requires a minimum number of azimuth samples for sparse scene reconstruction, and demonstrates its robustness to low sparse support similarity and additive observation noise. Simulation and experiment results clearly demonstrated the superiority of the proposed algorithm over existing clustered BCS, and multitask compressive sensing method.

\section{APPENDIX}

Derivation of Eq. (33)

According to the data-argumentation technique, we can acquire the analytical posterior distribution for the latent vector $\gamma$ in a hierarchical sampling scheme instead of directly sampling the vector $\gamma$ in the logistic model. Based on the joint likelihood in Eq. (23), we can acquire the posterior distribution of $\gamma$ as

$$
p(\gamma \mid \mathbf{q}, \mathbf{z}, \boldsymbol{\Sigma}) \propto \prod_{i=1}^{M} \operatorname{Bern}\left[1 /\left(1+e^{-\rho \gamma_{i}}\right)\right] \mathcal{N}(\gamma \mid 0, \boldsymbol{\Sigma}) .
$$

By utilizing Eqs. (31) and (32), we obtain,

$$
\begin{aligned}
p(\boldsymbol{\gamma} \mid \mathbf{q}, \mathbf{z}, \boldsymbol{\Sigma}) & \propto \prod_{i=1}^{M} \exp \left(\frac{z_{i} \rho \gamma_{i}}{2}-\frac{q_{i} \rho^{2} \gamma_{i}^{2}}{2}\right) \\
& \times \exp \left[\frac{\left(z_{i}-1\right) \rho \gamma_{i}}{2}-\frac{q_{i} \rho^{2} \gamma_{i}^{2}}{2}\right] \\
& \times \exp \left(-\frac{1}{2} \boldsymbol{\gamma}^{T} \boldsymbol{\Sigma}^{-1} \boldsymbol{\gamma}\right) \\
& =\mathcal{N}(\boldsymbol{\gamma} \mid \boldsymbol{\mu}, \boldsymbol{\Gamma}),
\end{aligned}
$$

where $\boldsymbol{\mu}$ and $\boldsymbol{\Gamma}$ are respectively given in (34) and (35).

\section{REFERENCES}

[1] I. G. Cumming and F. H. Wong, Digital Processing of Synthetic Aperture Radar Data: Algorithms and Implementation. Artech House, 2005.

[2] H. Griffiths and N. Long, "Television-based bistatic radar," Proc. IEE-Radar Sonar Navig., vol. 133, no. 7, pp. 649-657, 1986.

[3] H. D. Griffths and C. J. Baker, "Passive coherent location radar systems. part 1: Performance prediction," Proc. IEE-Radar Sonar Navig., vol. 152, no. 3, pp. 153-159, 2005.

[4] Y. D. Zhang and B. Himed, "Moving target parameter estimation and SFN ghost rejection in multistatic passive radar," in Proc. IEEE Radar Conf., (Ottawa, Canada), pp. 1-5, April 2013.

[5] B. Rigling, "Bistatic synthetic aperture radar," in N.J. Willis and H. Griffiths, Eds., Advances in Bistatic Radar, Scitech Publishing, pp. 320-431, 2007.

[6] M. Conti, F. Berizzi, M. Martorella, E. D. Mese, D. Petri, and A. Capria, "High range resolution multichannel DVB-T passive radar," IEEE Aerospace and Electronic Systems Mag., vol. 27, no. 10, pp. 37-42, 2012.

[7] X. Mao, Y. D. Zhang, and M. G. Amin, "Low-complexity sparse reconstruction for high-resolution multi-static passive SAR imaging," EURASIP J. Adv. Signal Process., vol. 2014, no. 104, pp. 1-12, 2014.

[8] S. Wacks and B. Yazici, "Passive synthetic aperture radar imaging of ground moving targets," in Proc. SPIE 8394, Algorithms for Synthetic Aperture Radar Imagery XIX, (Baltimore, MD), pp. 83940E-1-83940E-10, May 2012.

[9] Y. Wu and D. C. Munson, "Wide-angle ISAR passive imaging using smoothed pseudo Wigner-Ville-distribution," in Proc. IEEE Radar Conf., (Atlanta, GA), pp. 363-368, Dec. 2001.

[10] A. Paulose, High Radar Range Resolution with the Step Frequency Wwaveform. M.S. Thesis, Naval Postgraduate School, Monterey, CA, 1994.

[11] Q. Wu, M. Xing, C. Qiu, Z. Bao, and T. Yeo, "Focusing of tandem bistatic configuation data with range migration algorithm," IEEE Geosci. Remote Sens. Lett., vol. 8, no. 1, pp. 88-92, 2010.

[12] W. Jing, Q. Wu, M. Xing, and Z. Bao, "Image formation of wide-swath high resolution MIMO-SAR," J. System Simulation, vol. 16 , no. 3, pp. 38-44, 2008.

[13] T. G. Moore, B. W. Zuerndorfer, and E. C. Burt, "Enhanced imagery using spectral-estimation-based techniques," Lincoln Lab. J., vol. 10, no. 2, pp. 171-186, 1997.

[14] K. E. Olsen and K. Woodbridge, "Multiband passive bistatic DVB-T radar range resolution improvements and implications," in Proc. Int. Radar Symp., (Warsaw, Poland), pp. 28-30, Sept. 2012.

[15] L. Zhang, M. Xing, C. Qiu, J. Li, J. Sheng, Y. Li, and Z. Bao, "Resolution enhancement for inversed synthetic aperture radar imaging under low SNR via improved compressive sensing," IEEE Trans. Geosci. Remote Sens., vol. 48, no. 10, pp. 38243838, 2010.

[16] M. Cetin and W. C. Karl, "Feature-enhanced synthetic aperture radar image formation based on nonquadratic regularization," IEEE Trans. Image Proc., vol. 10, no. 4, pp. 623-631, 2001.

[17] J. H. G. Ender, "On compressive sensing applied to radar," Signal Proc., vol. 90, no. 5, pp. 1402-1414, 2010.

[18] A. Budillon, A. Evangelista, and G. Schirinzi, "Threedimensional SAR focusing from multipass signals using compressive sampling," IEEE Trans. Geosci. Remote Sens., vol. 49, no. 1, pp. 488-499, 2011.

[19] J. A. Tropp and A. C. Gilbert, "Signal recovery from partial information via orthogonal matching pursuit," IEEE Trans. Info. Theory, vol. 53, no. 12, pp. 4655-4666, 2007.

[20] R. Tibshirani, "Regression shrinkage and selection via the Lasso,” J. Royal. Statist. Soc., vol. 58, no. 1, pp. 267-288, 1996.

[21] M. Skolnik, Radar Handbook, Third Edition. McGraw-Hill, 2008.

[22] M. Yuan and Y. Lin, "Model selection and estimation in regression with grouped variables," J. Royal Statist. Soc. Series $B$, vol. 68, no. 1, pp. 49-67, 2006.

[23] L. Jacob, G. Obozinski, and J. Vert, "Group Lasso with overlap and graph Lasso," in Proc. Int. Conf. Machine Learning, (Montreal, Canada), pp. 433-440, Jun. 2009.

[24] S. Ji, Y. Xue, and L. Carin, "Bayesian compressive sensing," IEEE Trans. Signal Proc., vol. 56, no. 6, pp. 2346-2356, 2008.

[25] M. E. Tipping, "Sparse Bayesian shrinkage and selection learning and the relevance vector machine," J. Machine Learning Research, vol. 1, no. 9, pp. 211-244, 2001.

[26] Z. Zhang and B. D. Rao, "Sparse signal recovery with temporally correlated source vector using sparse Bayesian learning," IEEE J. Sel. Topics in Signal Proc., vol. 5, no. 5, pp. 912-926, 2011.

[27] S. Ji, D. Dunson, and L. Carin, "Multitask compressive sampling," IEEE Trans. Signal Proc., vol. 57, no. 1, pp. 92106, 2009. 
[28] S. D. Babacan, S. Nakajima, and M. N. Do, "Bayesian groupsparse modeling and variational inference," IEEE Trans. Signal Proc., vol. 62, no. 11, pp. 2906-2921, 2014.

[29] G. Oliveri, P. Rocca, and A. Massa, "A Bayesian compressive sampling-based inversion for imaging sparse scatterers," IEEE Trans. Geosci. Remote Sens., vol. 49, no. 10, pp. 3993-4006, 2013.

[30] M. Carlin, P. Rocca, G. Oliveri, F. Viani, and A. Massa, "Directions-of-arrival estimation through Bayesian compressive sensing strategies," IEEE Trans. Antennas Propagat., vol. 61, no. 7, pp. 3828-3838, 2013.

[31] Q. Wu, Y. D. Zhang, M. G. Amin, and B. Himed, "Spacectime adaptive processing and motion parameter estimation in multistatic passive radar using sparse Bayesian learning," IEEE Trans. Geosci. Remote Sens., (in press).

[32] L. Poli, G. Oliveri, F. Viani, and A. Massa, "MT-BCS-based microwave imaging approach through minimum-norm current expansion," IEEE Trans. Antennas Propagat., vol. 61, no. 9, pp. 4722-4732, Sept. 2013.

[33] G. Oliveri, E. T. Bekele, F. Robol, and A. Massa, "Sparsening conformal arrays through a versatile BCS-based method," IEEE Trans. Antennas Propagat., vol. 62, no. 4, pp. 1681-1689, Apr. 2014.

[34] M. G. Amin, Y. D. Zhang, and B. Jokanovic, "Timefrequency signature reconstruction from random observations using multiple measurement vectors," in Proc. IEEE ICASSP, (Florence, Italy), pp. 345-349, May 2014.

[35] Q. Wu, Y. D. Zhang, and M. G. Amin, "Continuous structure based Bayesian compressive sensing for sparse reconstruction of time-frequency distribution," in Proc. Int. Conf. Digital Signal Proc., (Hong Kong, China), pp. 831-836, Aug. 2014.

[36] Q. Wu, Y. D. Zhang, M. G. Amin, and B. Himed, "Multi-static passive radar SAR imaging based on Bayesian compressive sensing," in Proc. SPIE 9109, Compressive Sensing III, (Baltimore, MD), pp. 910902-1-910902-9, May 2014.

[37] Q. Wu, Y. D. Zhang, M. G. Amin, and B. Himed, "Complex multitask Bayesian compressive sensing," in Proc. IEEE ICASSP, (Florence, Italy), pp. 3375-3379, May 2014.

[38] S. Qin, Y. D. Zhang, Q. Wu, and M. G. Amin, "DOA estimation of nonparametric spreading spatial spectrum based on Bayesian compressive sensing exploiting intra-task dependency," in Proc. IEEE ICASSP, (Brisbane, Australia), pp. 2399-2403, April 2015.

[39] L. Wang, L. Zhao, G. Bi, C. Wan, and L. Yang, "Enhanced ISAR imaging by exploiting the continuity of the target scene," IEEE Trans. Geosci. Remote Sens., vol. 52, no. 9, pp. 57365750, 2014.

[40] L. Wang, L. Zhao, G. Bi, C. Wan, and L. Yang, "Sparse representation-based ISAR imaging using Markov random field," IEEE J. Sel. Topics in Appl. Earth Observ. Remote Sensing, (in press).

[41] Q. Wu, Y. D. Zhang, M. G. Amin, and B. Himed, "Mutli-task Bayesian compressive sensing exploiting intra-task dependency," IEEE Signal Proc. Lett., vol. 22, no. 4, pp. 430434, 2015.

[42] Q. Wu, Y. D. Zhang, F. Ahmad, and M. G. Amin, "Compressive sensing based high-resolution polarimetric through-the-wall radar imaging exploiting target characteristics," IEEE Antennas and Wireless Propagat. Lett., vol. 14, pp. 1043-1047, 2015.

[43] T. Peleg, Y. C. Eldar, and M. Elad, "Exploiting statistical dependencies in sparse representations for signal recovery," IEEE Trans. Signal Proc., vol. 60, no. 5, pp. 2286-2303, 2012.

[44] A. Drémeau, C. Herzet, and L. Daudet, "Boltzmann machine and mean-field approximation for structured sparse decompositions," IEEE Trans. Signal Proc., vol. 60, no. 7, pp. 3425-3438, 2012.

[45] J. Ziniel and P. Schniter, "Dynamic compressive sensing of time-varying signals via approximate message passing," IEEE Trans. Signal Proc., vol. 61, no. 21, pp. 5270-5284, 2012.
[46] Q. Wu, Y. D. Zhang, M. G. Amin, and B. Himed, "Structured Bayesian compressive sensing exploiting spatial location dependence," in Proc. IEEE ICASSP, (Brisbane, Australia), pp. 3831-3835, April 2015.

[47] C. Nolan and M. Cheney, "Synthetic aperture inversion," Inverse Probl., vol. 18, no. 1, pp. 221-235, 2002.

[48] C. E. Yarmanb, L. Wang, and B. Yazici, "Doppler synthetic aperture hitchhiker imaging," Inverse Probl., vol. 26, no. 6, pp. 1-26, 2010.

[49] L. Wang, C. E. Yarmanb, and B. Yazici, "Doppler-hitchhiker: A novel passive synthetic aperture radar using ultranarrowband sources of opportunity," IEEE Trans. Geosci. Remote Sens., vol. 49, no. 10, pp. 3521-3537, 2011.

[50] C. R. Berger, B. Demissie, J. Heckenbach, P. Willett, and S. Zhou, "Signal processing for passive radar using OFDM waveforms," IEEE J. Selected Topics in Signal Proc., vol. 4, no. 1, pp. 226-238, 2010.

[51] E. I. George and R. E. Mcculloch, "Variable selection via Gibbs sampling," J. American Statist. Assoc., vol. 88, no. 423, pp. 881889, 1993.

[52] T. J. Mitchell and J. J. Beauchamp, "Bayesian variable selection in linear regression," J. American Statist. Assoc., vol. 83, no. 404, pp. 1023-1032, 1988.

[53] L. Yu, H. Sun, J. P. Barbot, and G. Zheng, "Bayesian compressive sensing for cluster structured sparse signals," Signal Proc., vol. 92, no. 1, pp. 259-269, 2012.

[54] M. K. Titsias and M. Lázaro-Gredilla, "Spike and slab variational inference for multi-task and multiple kernel learning," in Proc. Adv. Neural Info. Proc. Syst., (Granada, Spain), pp. 2339-2347, Dec. 2011.

[55] C. M. Bishop, Pattern Recognition and Machine Learning. Springer, 2006.

[56] J. H. Albert and S. Chib, "Bayesian analysis of binary and polychotomous response data," J. American Statist. Assoc., vol. 88, no. 422, pp. 669-679, 1993.

[57] N. G. Polson, J. G. Scott, and J. Windle, "Bayesian inference for logistic models using Polya-Gamma latent variables," $J$. American Statist. Assoc., vol. 108, no. 504, pp. 1339-1349, 2013.

[58] W. R. Trench, "An algorithm for the inversion of finite toeplitz matrices," J. Assoc. Comput. Math., vol. 12, no. 3, pp. 515-522, 1964.

[59] S. Zohar, "Toeplitz matrix inversion: The algorithm of w. r. trench," J. Assoc. Comput. Math., vol. 16, no. 4, pp. 592-601, 1969.

[60] Y. Rubner, C. Tomasi, and L. J. Guibas, "The earth movers distance as a metric for image retrieval," Int. J. Computer Vision, vol. 40, no. 2, pp. 99-121, 2000.

[61] J. E.Palmer, H. A. H. amd S. J. Searle, and L. M. Davis, "DVBT passive radar signal processing," IEEE Trans. Signal Proc., vol. 61, no. 8, pp. 2116-2126, 2013.

[62] D. L. Donoho and J. Tanner, "Counting faces of randomly projected polytopes when the projection radically lowers dimensions," Technical Report, Department of Statistics, Stanford University, 2006.

[63] H. Zhou, Y. Li, and Y. Su, "Multi-aspect SAR imaging based on compressive sensing," China Science, vol. 43, no. 7, pp. 751760, 2012

[64] S. Brusch, S. Lehner, T. Fritz, M. Soccorsi, A. Soloviev, and B. V. Schie, "Ship surveillance with TerraSAR-X," IEEE Trans. Geosci. Remote Sens., vol. 49, no. 3, pp. 1092-1103, 2011.

[65] X. Xing, K. Ji, H. Zou, W. Chen, and J. Sun, "Ship classification in TerraSAR-X images with feature space based sparse representation," IEEE Geosci. Remote Sens. Lett., vol. 10, no. 6, pp. 1562-1566, 2013. 


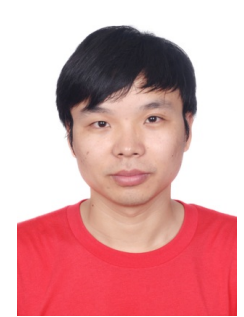

Qisong Wu (M'14) received his Ph.D. degree from the Xidian University, Xi'an, China, in 2010.

$\mathrm{He}$ is an Associate Professor with the Key Laboratory of Underwater Acoustic Signal Processing of Ministry of Education, Southeast University, Nanjing, China. He was a Post-Doctoral Associate with the Duke University, Durham, NC, USA from 2010 to 2013 and with the Villanova University, Villanova, PA, USA, from 2013 to 2015. His research interests include passive radar signal processing, sonar signal processing, array signal processing, sparse Bayesian learning, through-the-wall radar imaging, and synthetic aperture radar imaging.

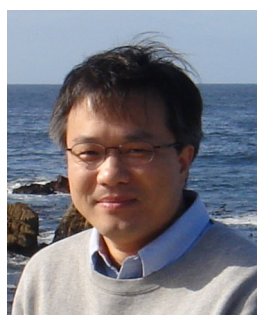

Yimin D. Zhang (SM'01) received his Ph.D. degree from the University of Tsukuba, Tsukuba, Japan, in 1988.

Dr. Zhang joined the faculty of the Department of Radio Engineering, Southeast University, Nanjing, China, in 1988. He served as a Director and Technical Manager at the Oriental Science Laboratory, Yokohama, Japan, from 1989 to 1995, a Senior Technical Manager at the Communication Laboratory Japan, Kawasaki, Japan, from 1995 to 1997, and a Visiting Researcher at the ATR Adaptive Communications Research Laboratories, Kyoto, Japan, from 1997 to 1998 . He was with the Villanova University, Villanova, PA, from 1998 to 2015, where he was a Research Professor with the Center for Advanced Communications. Since August 2015, he has been with the Department of Electrical and Computer Engineering, College of Engineering, Temple University, Philadelphia, PA, where he is currently an Associate Professor. His general research interests lie in the areas of statistical signal and array processing applied for radar, communications, and navigation, including compressive sensing, convex optimization, time-frequency analysis, MIMO system, radar imaging, target localization and tracking, wireless networks, and jammer suppression. He has published more than 270 journal articles and conference papers and 11 book chapters.

Dr. Zhang is a member of the Sensor Array and Multichannel Technical Committee of the IEEE Signal Processing Society. He is an Associate Editor for the IEEE Transactions on Signal Processing, and serves on the Editorial Board of the Signal Processing journal. He was an Associate Editor for the IEEE Signal Processing Letters during 2006-2010, and an Associate Editor for the Journal of the Franklin Institute during 2007-2013.

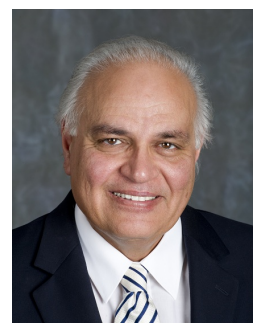

Moeness G. Amin (F'01) received his Ph.D. degree in Electrical Engineering from University of Colorado in 1984.

He has been on the Faculty of the Department of Electrical and Computer Engineering at Villanova University since 1985 . In 2002, he became the Director of the Center for Advanced Communications, College of Engineering. He is a Fellow of the Institute of Electrical and Electronics Engineers (IEEE), 2001; Fellow of the International Society of Optical Engineering, 2007; and a Fellow of the Institute of Engineering and Technology (IET), 2010. Dr. Amin is a Recipient of the IEEE Third Millennium Medal, 2000; Recipient of the 2014 IEEE Signal Processing Society Technical Achievement Award; 2015 IEEE Warren D. White Award for Excellence in Radar Engineering; Recipient of the 2009 Individual Technical Achievement Award from the European Association of Signal Processing; Recipient of the 2010 NATO Scientific Achievement Award; Recipient of the Chief of Naval Research Challenge Award, 2010; Recipient of Villanova University Outstanding Faculty Research Award, 1997; and the Recipient of the IEEE Philadelphia Section Award, 1997. He was a Distinguished Lecturer of the IEEE Signal Processing Society, 20032004, and is currently the Chair of the Electrical Cluster of the Franklin Institute Committee on Science and the Arts. Dr. Amin has over 700 journal and conference publications in the areas of Wireless Communications, Time-Frequency Analysis, Sensor Array Processing, Waveform Design and Diversity, Interference Cancellation in Broadband Communication Platforms, Satellite Navigations, Target Localization and Tracking, Direction Finding, Channel Diversity and Equalization, Ultrasound Imaging and Radar Signal Processing. He co-authored 18 book chapters. He is the Editor of the two books Through the Wall Radar Imaging and Compressive Sensing for Urban Radar, published by CRC Press in 2011 and 2014, respectively.

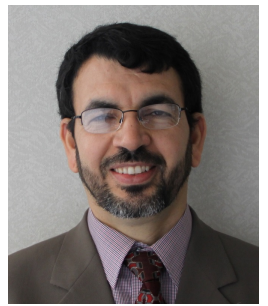

Braham Himed (S'88-M'90-SM'01-F'07) received his Engineer Degree in electrical engineering from Ecole Nationale Polytechnique of Algiers in 1984, and his M.S. and Ph.D. degrees both in electrical engineering, from Syracuse University, Syracuse, NY, in 1987 and 1990, respectively.

Dr. Himed is a Technical Advisor with the Air Force Research Laboratory, Sensors Directorate, RF Technology Branch, in Dayton Ohio, where he is involved with several aspects of radar developments. His research interests include detection, estimation, multichannel adaptive signal processing, time series analyses, array processing, adaptive processing, waveform diversity, MIMO radar, passive radar, and over the horizon radar. Dr. Himed is the recipient of the 2001 IEEE region I award for his work on bistatic radar systems, algorithm development, and phenomenology. He is a Fellow of the IEEE and the Vice-Chair of the AESS Radar Systems Panel. He is the recipient of the 2012 IEEE Warren White award for excellence in radar engineering. Dr. Himed is also a Fellow of AFRL (Class of 2013). 